\title{
Dichotomous metabolic networks govern human ILC2 proliferation and function
}

\author{
Laura Surace ${ }^{\circledR 1}$, Jean-Marc Doisne ${ }^{\circledR 1}$, Carys A. Croft ${ }^{11,2}$, Anna Thaller,2, Pedro Escoll ${ }^{3}{ }^{3}$, \\ Solenne Marie', Natalia Petrosemoli ${ }^{4}$, Vincent Guillemot ${ }^{4}$, Valerie Dardalhon ${ }^{5}$, Davide Topazio ${ }^{6}$, \\ Antonia Cama7, Carmen Buchrieser ${ }^{1}{ }^{3}$, Naomi Taylor ${ }^{5}$, Ido Amit ${ }^{8}{ }^{8}$, Olimpia Musumeci ${ }^{9}$ and \\ James P. Di Santo ${ }^{1 \times}$
}

\begin{abstract}
Group 2 innate lymphoid cells (ILC2s) represent innate homologs of type 2 helper $T$ cells $\left(T_{\mathrm{H}} 2\right)$ that participate in immune defense and tissue homeostasis through production of type 2 cytokines. While T lymphocytes metabolically adapt to microenvironmental changes, knowledge of human ILC2 metabolism is limited, and its key regulators are unknown. Here, we show that circulating 'naive' ILC2s have an unexpected metabolic profile with a higher level of oxidative phosphorylation (OXPHOS) than natural killer (NK) cells. Accordingly, ILC2s are severely reduced in individuals with mitochondrial disease (MD) and impaired OXPHOS. Metabolomic and nutrient receptor analysis revealed ILC2 uptake of amino acids to sustain OXPHOS at steady state. Following activation with interleukin-33 (IL-33), ILC2s became highly proliferative, relying on glycolysis and mammalian target of rapamycin (mTOR) to produce IL-13 while continuing to fuel OXPHOS with amino acids to maintain cellular fitness and proliferation. Our results suggest that proliferation and function are metabolically uncoupled in human ILC2s, offering new strategies to target ILC2s in disease settings.
\end{abstract}

ILCs have important roles in systemic as well as local tissue immunity and are involved in early immune responsiveness and regenerative processes that restore homeostasis ${ }^{1-3}$. Five ILC subsets (ILC1, ILC2, ILC3, lymphoid tissue inducer cells (LTi) and NK cells) have been described both in mice and humans based on their transcription factor dependency and signature cytokine production ${ }^{4-10}$. ILC2s rely on the transcription factor GATA-3 for development and for regulation of the expression of type 2 cytokines, including IL-5, IL-9 and IL-13, and amphiregulin in response to alarmins, such as IL-33 (refs. ${ }^{11-15}$ ). ILC2s reside mostly in barrier tissues; however, they can also enter the circulation and traffic between tissues ${ }^{16-20}$. In mice, two subsets have been identified: the tissue-resident natural ILC2s (nILC2s) and the inflammatory ILC2s (iILC2s), which are generated following the induction of type 2 inflammation in response to IL-25 and have the capacity to migrate ${ }^{21-23}$. Whether this dichotomy exists in humans is still a matter of debate. Human circulating ILC2s are described as CD45RA ${ }^{+}$naive cells, which reside in a resting state and are then recruited into tissues to become activated $^{17,24}$. Circulating and tissue-resident human ILC2s have heterogeneous phenotypes and functions $s^{1,7,25}$, but their key regulators remain poorly defined.

Mitochondria play a central role in cellular metabolism and are integral to a functional immune response ${ }^{26}$. Immunometabolism studies on $\mathrm{T}$ cells have defined specific metabolic programs (glycolysis and OXPHOS) following activation and differentiation ${ }^{27-31}$. Metabolite tracing revealed that activated $\mathrm{T}$ cells are preferentially glycolytic and take up glutamine to replenish tricarboxylic acid (TCA) cycle intermediates during cell proliferation and cytokine production $^{32}$. By contrast, memory T cells exhibit a metabolic switch to fatty acid (FA) metabolism, mitochondria fusion and increased respiration ${ }^{33-35}$. However, single-cell metabolic flux analysis revisited this concept of a direct naive-to-activated $\mathrm{T}$ cell metabolic switch, showing that naive $\mathrm{T}$ cells are metabolically heterogeneous and explore 'metabolic checkpoints' before engaging into a specific program, which would then dictate downstream function ${ }^{30}$. While ILC2s have been reported to rely on nutrients other than glucose, including $\mathrm{FA}^{36,37}$ and arginine ${ }^{38}$, it is not known how different metabolic identities in ILC2s are generated following activation and linked to their biological roles. Here, we show that circulating naive human ILC2s reside in a highly active metabolic state relying on branched chain amino acids (BCAAs) and arginine to support mitochondrial OXPHOS. Following activation, ILC2s leverage their enhanced amino acid metabolism for rapid proliferation but engage glycolysis for effector cytokine production. The independent regulation of proliferation and effector function by dichotomous metabolic pathways opens avenues for manipulating ILC2s in disease settings.

We used MitoTracker Green FM and tetramethylrhodamine (TMRM) staining as described in ref. ${ }^{39}$ to assess mitochondrial mass and membrane polarization $\left(\Delta \psi_{\mathrm{m}}\right)$ in human blood ILC2s isolated from healthy donors. $\mathrm{CD} 56^{+} \mathrm{CD} 16^{+} \mathrm{NK}^{\mathrm{dim}}$ cells (Extended Data Fig. 1a,b) were used as an ILC reference for comparison. Previous studies suggested that blood ILC2s were naive ${ }^{17,24}$; however, fluorescence-activated cell sorting (FACS) analysis revealed that they have two times the mitochondrial mass of $\mathrm{NK}^{\mathrm{dim}}$ cells (Fig. 1a). Despite heterogeneity across donors (Extended Data Fig. 1c), TMRM intensity and TMRM/MitoTracker ratios were consistently higher in ILC2s at steady state (Fig. 1a). As mitochondria fission and

IInnate Immunity Unit, Institut Pasteur, Inserm U1223, Paris, France. ${ }^{2}$ Université de Paris, Sorbonne Paris Cité, Paris, France. ${ }^{3}$ Biology of Intracellular Bacteria Unit, Institut Pasteur, CNRS UMR 3525, Paris, France. "Bioinformatics and Biostatistics Hub, Center of Bioinformatics, Biostatistics, and Integrative Biology, Institut Pasteur, Paris, France. Institut de Génétique Moléculaire de Montpellier, University of Montpellier, CNRS, Montpellier, France. ${ }^{6}$ Department of Otolaryngology, Hospital 'Mazzini', Teramo, Italy. ${ }^{7}$ Department of Maxillofacial and Otolaryngology, Hospital 'F. Renzetti', Lanciano, Italy. ${ }^{8}$ Department of Immunology, Weizmann Institute of Science, Rehovot, Israel. 'Unit of Neurology and Neuromuscular Disorders, Department of Clinical and Experimental Medicine, University of Messina, Messina, Italy. $₫ e$-mail: james.di-santo@pasteur.fr 
fusion are associated with glycolysis and OXPHOS, respectively ${ }^{40}$, we studied mitochondrial morphology and confirmed that ILC2s have higher mitochondrial mass and polarization than that observed in NK cells and they present fused mitochondria (Extended Data Fig. 1d,e). Because of their relative scarcity in blood, we could not monitor real-time ILC2 oxygen consumption or glycolytic rates. Instead, we measured ATP and ADP by mass spectrometry. We found that circulating ILC2s have high cytosolic ATP/ADP ratios compared to that observed in $\mathrm{NK}^{\mathrm{dim}}$ cells (Extended Data Fig. 1f and Supplementary Data 1), consistent with enhanced mitochondrial function and inhibition of glycolysis. Memory T cells have highly polarized mitochondria and enhanced OXPHOS compared to naive T cells ${ }^{27-41}$. When comparing TMRM and MitoTracker patterns in ILC2s versus in naive and central/effector memory $\mathrm{CD} 4^{+} \mathrm{T}$ cell subsets (Extended Data Fig. 2a), ILC2s showed a distinct mitochondrial signature, suggesting exposure to environmental signals.

We next assessed the bioenergetic profile of tissue ILC2s. Despite their scarcity (Extended Data Fig. 2b), tonsil ILC2s appeared to have similar phenotypic and functional profiles as their blood counterparts ${ }^{27}$. Tonsil ILC2s (detected as $\mathrm{Lin}^{-} \mathrm{CD} 127^{+} \mathrm{CD} 161^{+} \mathrm{GATA}-3^{+} \mathrm{ST} 2^{+}$ cells; Extended Data Fig. 2c-e) showed similar mitochondria polarization and mass as blood ILC2s (Extended Data Fig. 2f), suggesting a close metabolic relationship. One explanation is that some tonsil ILC2s may be blood derived and not (yet) affected metabolically by local environmental signals, which could include IL-2, IL-7, IL-33 and/or other cytokines.

Individuals with MDs (impaired OXPHOS) have a twofold reduction in frequencies of blood $\mathrm{NK}^{\mathrm{dim}}$ cells and a preferential loss of long-lived 'memory-like' $\mathrm{NK}^{\mathrm{dim}}$ cells ${ }^{39}$. Reductions in blood ILC2s in individuals with MDs were even more profound (about 80\%) (Fig. 1b and Extended Data Fig. 3a), while other lymphoid subsets $\left(\mathrm{CD}^{+}\right.$and $\mathrm{CD} 8^{+} \mathrm{T}$ cells) were less affected (Extended Data Fig. 3b). Interestingly, residual ILC2s in individuals with MDs showed decreased GATA-3 expression (Fig. 1c), decreased mitochondrial mass and polarization (Fig. 1d) and more Annexin- $\mathrm{V}^{+}$ cells (Extended Data Fig. 3c), suggesting a requirement for mitochondrial function in ILC2 homeostasis.

As defects in components of the mitochondrial ETC underlie MDs in affected individuals (Supplementary Table 1), we corroborated these findings by exposing blood ILC2s to ETC inhibitors (Extended Data Fig. 3d,e). Inhibition of complexes I (rotenone) and III (antimycin A) significantly decreased the percentage of MitoTracker ${ }^{+}$TMRM $^{+}$cells (Fig. 1e,f) and impaired membrane potential, but not mitochondrial mass, in both ILC2s and $\mathrm{NK}^{\mathrm{dim}}$ cells (Fig. $1 \mathrm{~g}$ and Extended Data Fig. 3e). We observed that rotenone and antimycin A, but not complex II inhibition (TTFA), strongly impaired ILC2 survival, whereas $\mathrm{NK}^{\text {dim }}$ cells were largely unaffected (Fig. 1h). These results further support the essential role for active ETC complex I and complex III for survival of blood ILC2s.

We next purified ILC2s and analyzed the cellular metabolome using high-resolution mass spectrometry (Supplementary Data 1). Pathway analysis revealed an enrichment in metabolites from arginine, BCAAs (valine, leucine/isoleucine) and glutamine metabolism (Extended Data Fig. 4a). Valine and isoleucine were the most abundant amino acids in circulating ILC2s, followed by arginine and glutamate (Fig. 2a). Arginine and glutamine/glutamate have been described to play a role in lymphocyte proliferation ${ }^{38,41,42}$, while BCAAs are essential amino acids required for mTOR activation ${ }^{43}$. We detected enhanced expression of SLC3A2 (light subunit of the LAT1 receptor) and SLC43A2 (LAT4) in ILC2s (Fig. 2b). LAT1 and LAT4 preferentially transport BCAAs. Steady-state blood ILC2s showed high surface expression of CD98 (LAT1) but not GLUT1 (glucose transporter) or CD36 (FA transporter). ASCT2 (glutamine transporter) was also highly expressed (Fig. 2c), providing an explanation for the high glutamate levels observed in circulating ILC2s (Fig. 2c). We found a similar pattern of nutrient transporter expression on tonsil ILC2s (Extended Data Fig. 4b).

Pharmacological inhibition of glycolysis (2DG), arginase-1 (nor-NOHA), glutamine conversion into glutamate (BPTES) and BCAA transaminase that converts BCAA into glutamate (BCATi) (Fig. 2d) did not impact ILC2 cell number at the concentration used (Extended Data Fig. 4c). However, inhibition of arginase-1 and BCAT resulted in a reduced percentage of MitoTracker ${ }^{+} \mathrm{TMRM}^{+}$ ILC2s (Fig. 2e,f), with a significant reduction in mitochondrial $\Delta \psi_{\mathrm{m}}$ (Extended Data Fig. 4d). The TMRM-to-MitoTracker ratio was also decreased (Fig. 2g), suggesting an impact on OXPHOS more than mitochondria remodeling or biogenesis. Given the observed heterogeneity in freshly sorted ILC2s (Extended Data Fig. 1c), we analyzed individual ILC2 TMRM profiles after treatment with OXPHOS inhibitors. Responses were uniform (Extended Data Fig. 4e), suggesting that the dependence of circulating ILC2s on OXPHOS/ oxygen consumption and amino acids represents a fundamental property required to sustain mitochondrial activity. Corroborating these findings, we found upregulation of enzymes involved in the TCA cycle but low expression of enzymes involved in glycolysis and FA oxidation (Extended Data Fig. 5 and Supplementary Data 2). We also observed upregulation of enzymes involved in the conversion of BCAAs into glutamate and acetyl-CoA as well as upregulation of arginase- 1 and ornithine aminotransferase, consistent with the role of BCAAs and arginine in fueling the TCA cycle (Extended Data Fig. 5) also described in differentiated $\mathrm{T}$ cells ${ }^{44}$. Together, our data show that circulating ILC2s are not in a resting metabolic state but already show elevated profiles characterized by increased BCAA and arginine levels that sustain OXPHOS required for cell fitness and survival.

To gain insight into the metabolic changes that circulating ILC2s undergo following exposure to tissue-derived cytokines, we cultured sorted blood ILC2s with IL-2 and IL-7, which support basal proliferation without activation ${ }^{11,45}$, or with IL-2, IL-7 and IL-33 that can fully activate ILC2s with robust proliferation and secretion of type 2 cytokines $^{11,45,46}$. Circulating ILC2s are $\mathrm{CRTH} 2^{+} \mathrm{ST} 2^{-}$(Extended Data Fig. 6a), and a combination of IL-2 and IL-7 upregulated the IL-33 receptor (ST2) (Extended Data Fig. 6b,c), allowing IL-33 to exert its effects. Accordingly, IL-33 alone (or the combinations

Fig. 1 | Complex I and III of the electron transport chain (ETC) support ILC2 survival. a, TMRM and MitoTracker measurement in freshly sorted ILC2s and $\mathrm{NK}^{\text {dim }}$ cells. Representative plots show MitoTracker and TMRM geometric mean fluorescence intensity (gMFI) and the ratio between the gMFI of TMRM and MitoTracker $(n=7)$. b-d, Comparison of healthy donors (HD; black) and individuals with MD (PT; green). Representative plots show the percentage of ILC2s in live CD45+ cells (b), GATA-3 gMFI in circulating ILC2s (c) (HD =7; PT =7) and MitoTracker and TMRM gMFI and the ratio between the geometric mean of TMRM and MitoTracker $(\mathbf{d})(\mathrm{HD}=5 ; \mathrm{PT}=4)$. e-h, Freshly sorted ILC2s and NK ${ }^{\text {dim }}$ cells were cultured without additional cytokines for $18 \mathrm{~h}$ in DMSO, rotenone $(1 \mu \mathrm{M})$, antimycin $\mathrm{A}(1 \mu \mathrm{M})$, 2-thenoyltriflouroacetone (TTFA; $1 \mu \mathrm{M})$ or oligomycin $(1 \mu \mathrm{M})$. Representative TMRM and MitoTracker FACS plots (e), percentage of MitoTracker ${ }^{+} \mathrm{TMRM}^{+}$cells in live $\mathrm{CD}^{4} 5^{+}$cells $(\mathbf{f})$, the ratio between the geometric mean of TMRM and MitoTracker ( $\mathbf{g}$ ) and cell counts calculated by FACS $(\mathbf{h})$ are shown $(n=5)$. The data in $\mathbf{a}$ are representative of five independent experiments with two to five donors each. Each dot in $\mathbf{b}$ represents one donor. The data in $\mathbf{c}$ and $\mathbf{d}$ are representative of two independent experiments with two to five healthy donors and two to four individuals with MD. The data in $\mathbf{e}-\mathbf{h}$ are representative of three independent experiments with at least three donors each. Floating bars in $\mathbf{a}-\mathbf{h}$ indicate the mean, minimum and maximum values within the dataset. Statistics were assessed by one-way analysis of variance (ANOVA) with a Dunnett correction; NS, not significant $(P>0.05) ;{ }^{\star} P<0.05 ;{ }^{\star \star} P<0.01 ;{ }^{\star \star \star} P<0.001 ;{ }^{\star \star \star \star} P<0.0001$. 
of IL-2 and IL-33 or IL-7 and IL-33) did not promote ILC2 proliferation or mitochondria polarization (Extended Data Fig. 6d,e). As expected, basal proliferation in cells treated with IL-2 and IL-7 was enhanced by IL-33 (Fig. 3a), and production of type 2 cytokines was clearly induced (Extended Data Fig. 6f). ILC2s stimulated with IL-2, IL-7 and IL-33 showed an increase in mitochondrial $\Delta \psi_{\mathrm{m}}$ a
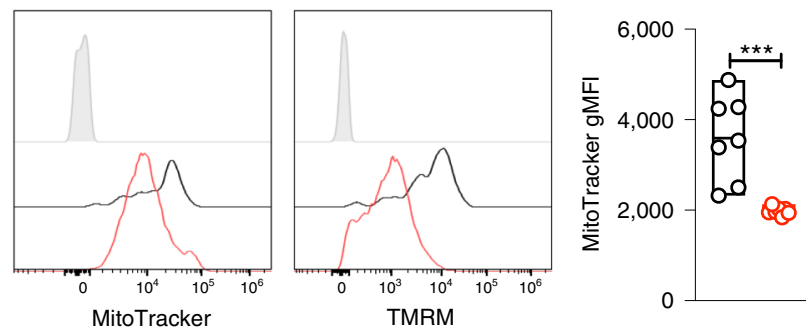

b
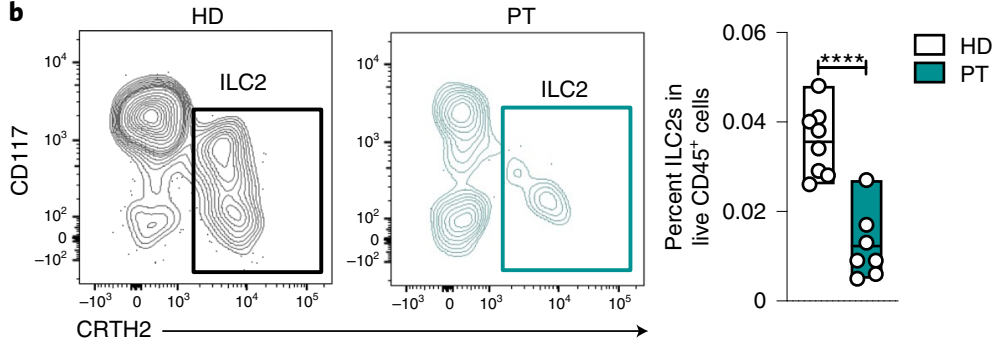

c

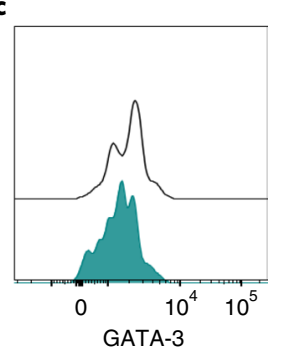

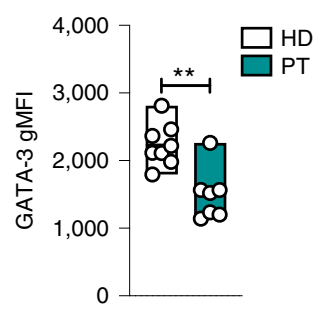

d
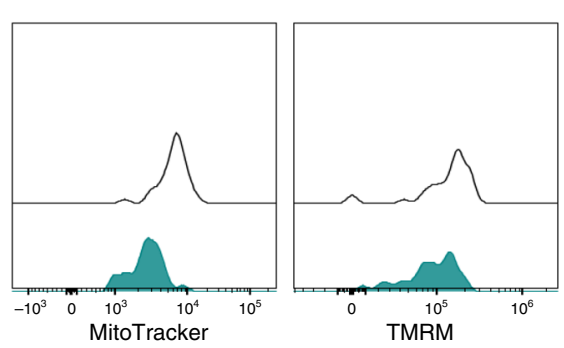

e
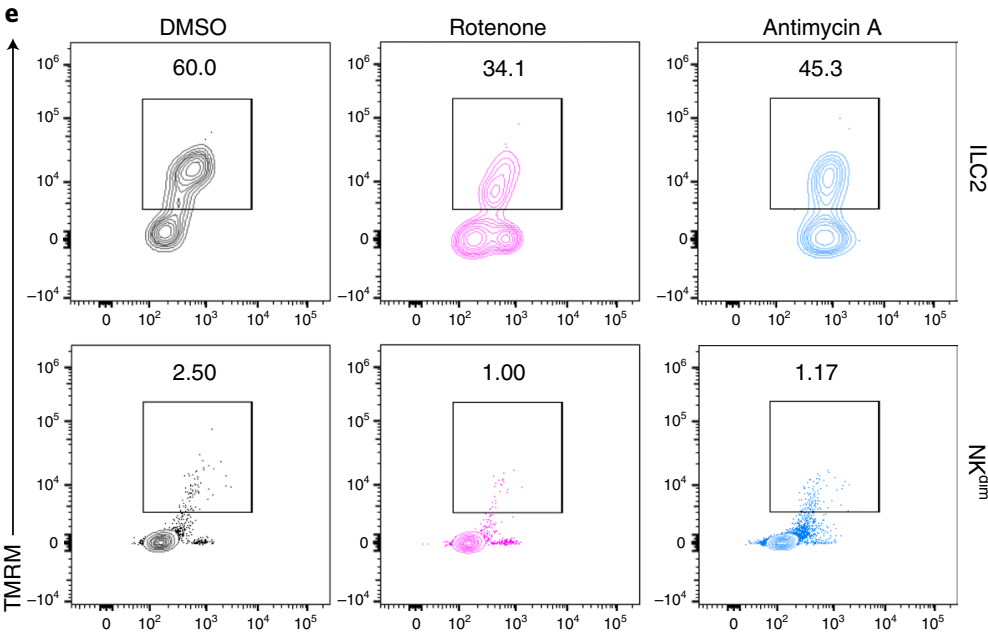

MitoTracker

h

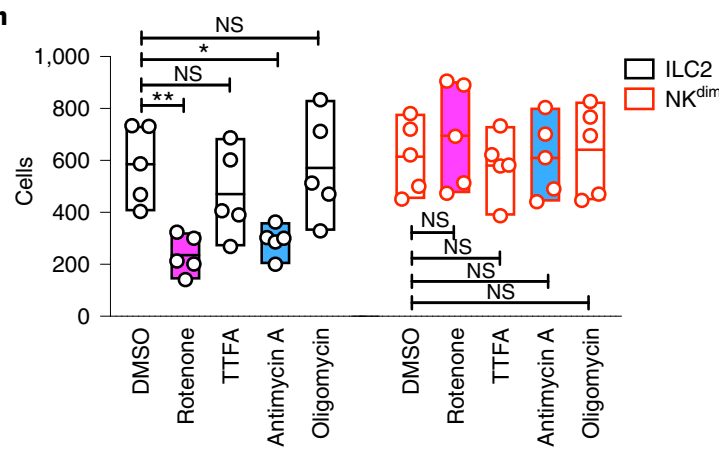

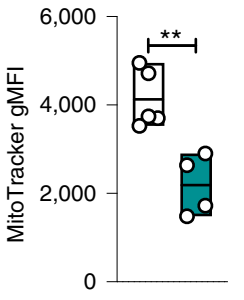
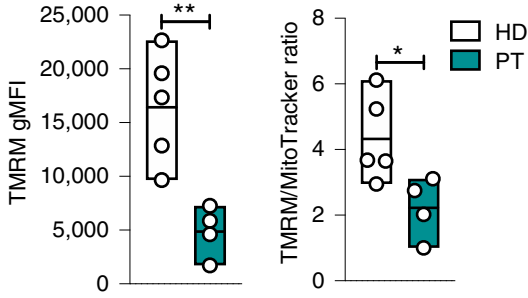
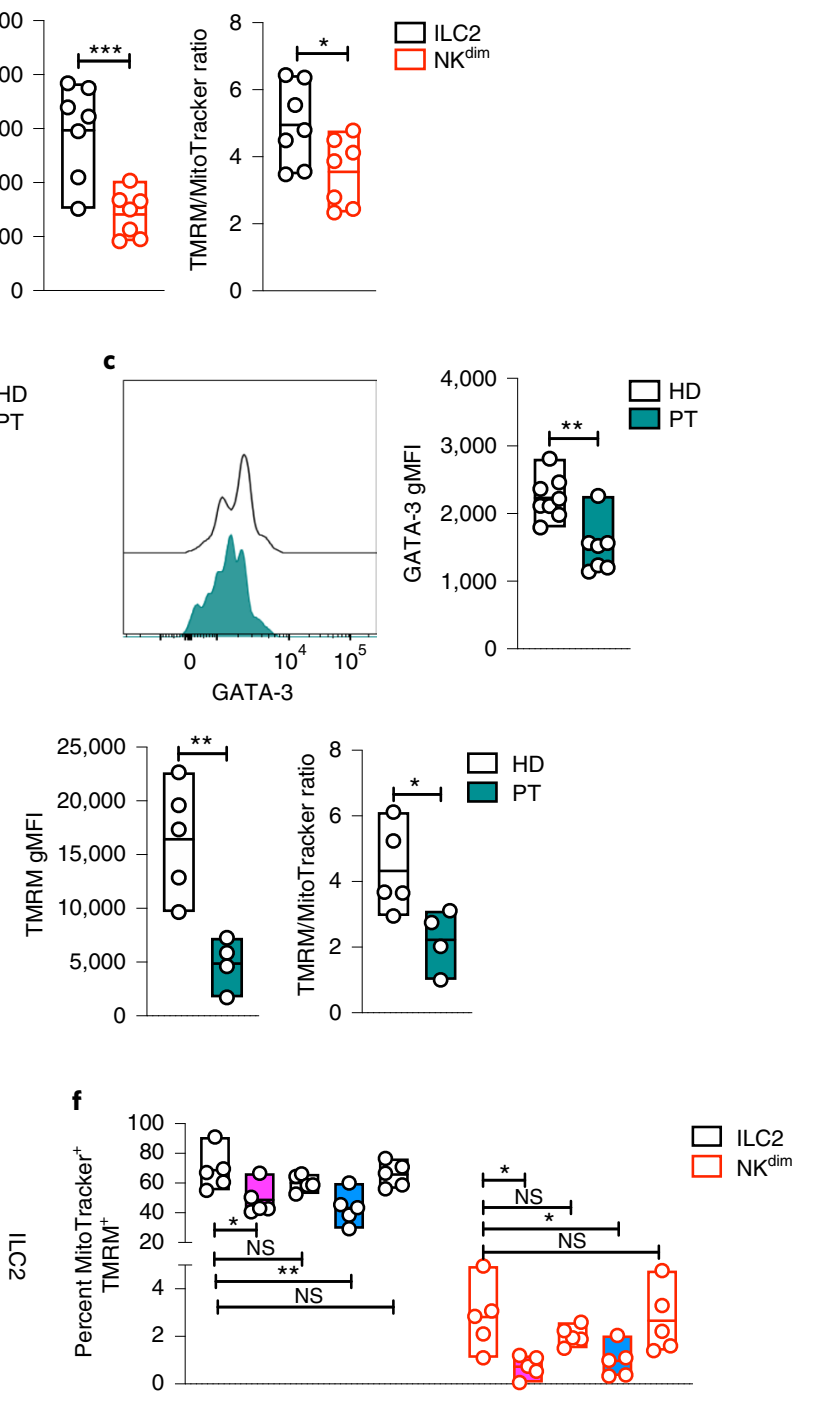

$\mathbf{g}$

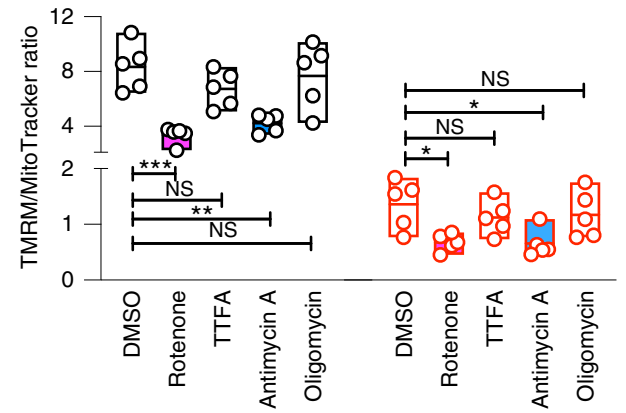


a

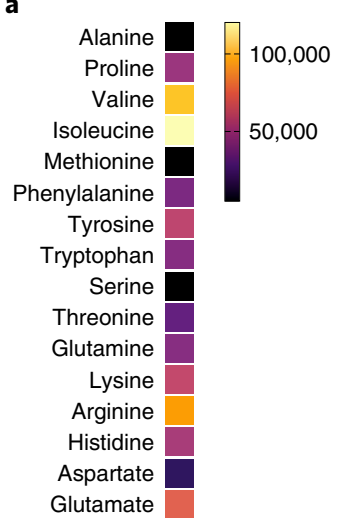

b

b
SLC1A5/ASCT2
SLC3A2/LAT1_IS
SLC7A5/LAT1_hs
SLC7A6/LAT2
SLC43A1/LAT3
SLC43A2/LAT4
SLC7A1/CAT1
SLC27A1/FATP1
SLC27A2/FATP2
SLC27A3/FATP3
SLC27A4/FATP4
SLC27A5/FATP5
CD36/FAT
SLC2A1/GLUT1
20

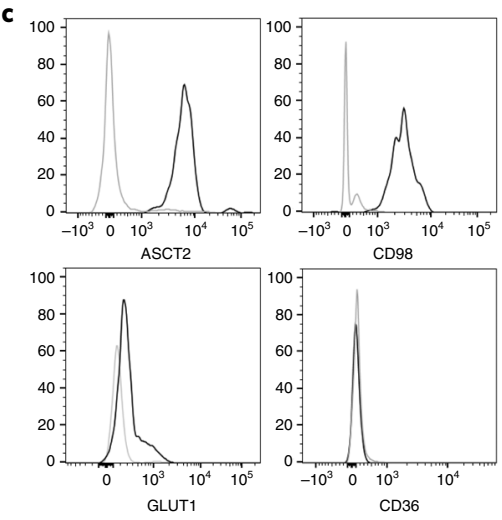

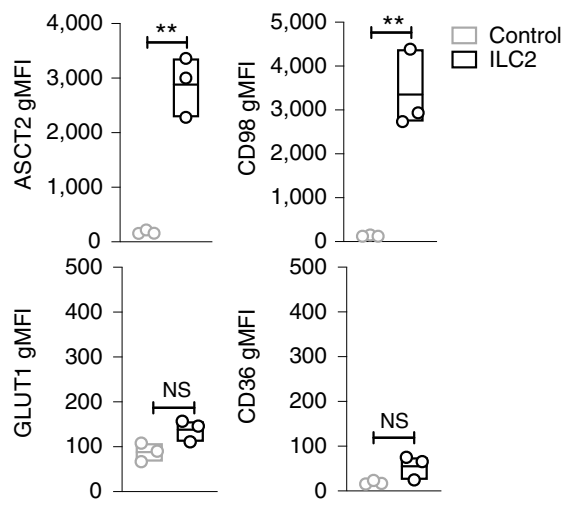
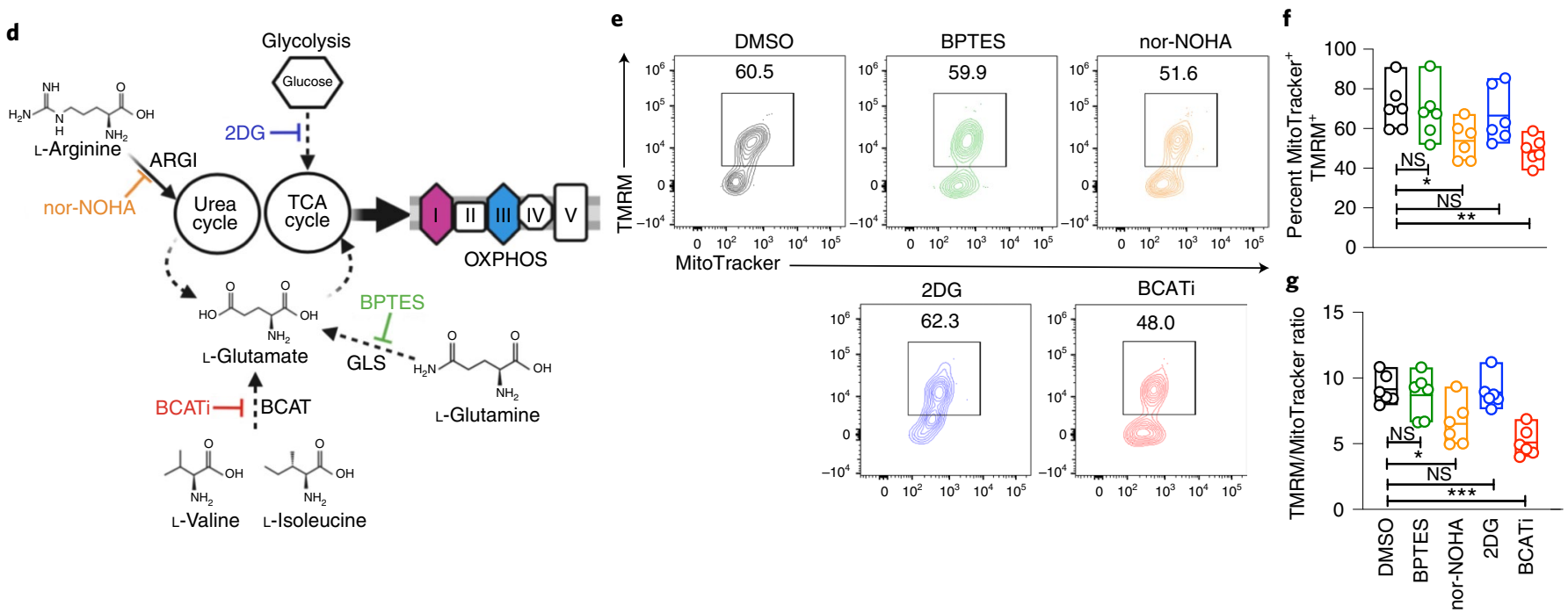

Fig. 2 | BCAA and arginine metabolism fuel OXPHOS in steady-state ILC2s. a, Heat map of amino acid intensities quantified by label-free mass spectrometry in freshly sorted ILC2s. b, Nutrient receptor analysis in fresh ILC2s by RNA sequencing (RNA-seq) (median value among three donors; relative expression). c, Representative plots of ASCT2 (glutamine transporter), CD98 (LAT1; large amino acid transporter), GLUT1 (glucose transporter) and CD36 (FA receptor) and MFI quantification of each receptor versus isotype control $(n=3)$. $\mathbf{d}-\mathbf{g}$, Freshly sorted ILC2s were cultured without additional cytokines for $18 \mathrm{~h}$ in DMSO, $\mathrm{N}^{\omega}$-hydroxy-nor-arginine (nor-NOHA; $\left.1 \mu \mathrm{M}\right), \mathrm{BCAA}$ transferase inhibitor (BCATi; $\left.1 \mu \mathrm{M}\right)$, bis-2-(5-phenylactamido1,3,4-thiadiazol-2-yl)ethyl sulfide (BPTES; $1 \mu \mathrm{M}$ ) or 2-deoxy-D-glucose (2DG; $1 \mu \mathrm{M}$ ) to selectively inhibit the pathways depicted in the schematic in d; ARGI, arginase-1; GSL, glutaminase. A representative TMRM and MitoTracker FACS plot in ILC2s (e), the percentage of MitoTracker ${ }^{+}$TMRM $^{+}$cells $(\mathbf{f})$ and the ratio between TMRM and MitoTracker MFI $(\mathbf{g})(n=6)$ are shown. The data in a are summarized from four donors, and a minimum of two technical replicates were analyzed per run. Data in $\mathbf{b}$ were extracted from an RNA-seq dataset, and the median was calculated from the values of three healthy donors. Data in $\mathbf{c}$ are representative of three independent experiments with at least three donors each. Data in $\mathbf{d}-\mathbf{g}$ are summarized from two independent experiments with at least two donors, and plots are representative of a total of four independent experiments. The floating bars in $\mathbf{c}, \mathbf{f}$ and $\mathbf{g}$ indicate the mean, minimum and maximum values within the dataset. Statistics were assessed by two-tailed $t$-test (c) and one-way ANOVA with Dunnett correction (f and $\mathbf{g})$; NS, not significant $(P>0.05) ;{ }^{\star} P<0.05 ;{ }^{\star \star} P<0.01 ;{ }^{\star \star \star} P<0.001$.

and a slight decrease in mitochondrial mass (Fig. 3b and Extended Data Fig. 6g). Analysis of oxygen consumption rate (OCR) showed that IL-33 increased ILC2 basal and maximal respiration (Fig. 3c and Extended Data Fig. 6h) as well as the spare respiratory capacity (SRC) compared to cells stimulated in IL-2 and IL-7 (Fig. 3d). Together, these results indicate that IL-33-activated ILC2s show enhanced OXPHOS and maintain high cellular fitness despite stresses associated with elevated metabolic activity.

We next cultured ILC2s with or without IL-33 under hypoxic conditions (3\% oxygen). We observed that hypoxia led to a decrease in ILC2 survival as well as a loss of GATA-3 and ST2 protein (both gMFI and percentage of positive cells; Fig. $3 \mathrm{e}-\mathrm{g}$ and Extended Data Fig. 7a). We did not find significant changes in the expression of other ILC2-related proteins or more general lymphoid markers (Extended Data Fig. 7a), suggesting that hypoxia regulates a specific program centered around GATA-3 and ST2. As previously reported, the HIF-1 $\alpha-P K M$ axis modulates murine ILC2 bioenergetic balance and IL-33 responsiveness ${ }^{47}$. We found enhanced expression of HIF-1 $\alpha$ (Extended Data Fig. 7b) and PKM concomitant with a reduction in GATA-3 and ST2 transcription (Extended Data Fig. 7c) in IL-33-stimulated ILC2s under hypoxic conditions, confirming this HIF- $1 \alpha-$ PKM-ST2 axis in human ILC2s. We further observed that inhibition of ETC complexes I and III impaired survival and accentuated apoptosis of cytokine-activated ILC2s (Fig. 3h and Extended Data Fig. 7d), resulting in a loss of mitochondrial membrane potential and mass (Fig. $3 \mathrm{i}$ and Extended Data Fig. 7e). Together, these results confirm the crucial role of ETC function and oxygen in the homeostasis of cytokine-activated ILC2s.

IL-33 stimulated ILC2s to increase their glycolytic capacity (Fig. 3j) while maintaining elevated OXPHOS and SRC (Fig. 3c,d). We hypothesized that ILC2s should rely on different nutrient sources to support their bioenergetic settings. Comparison 

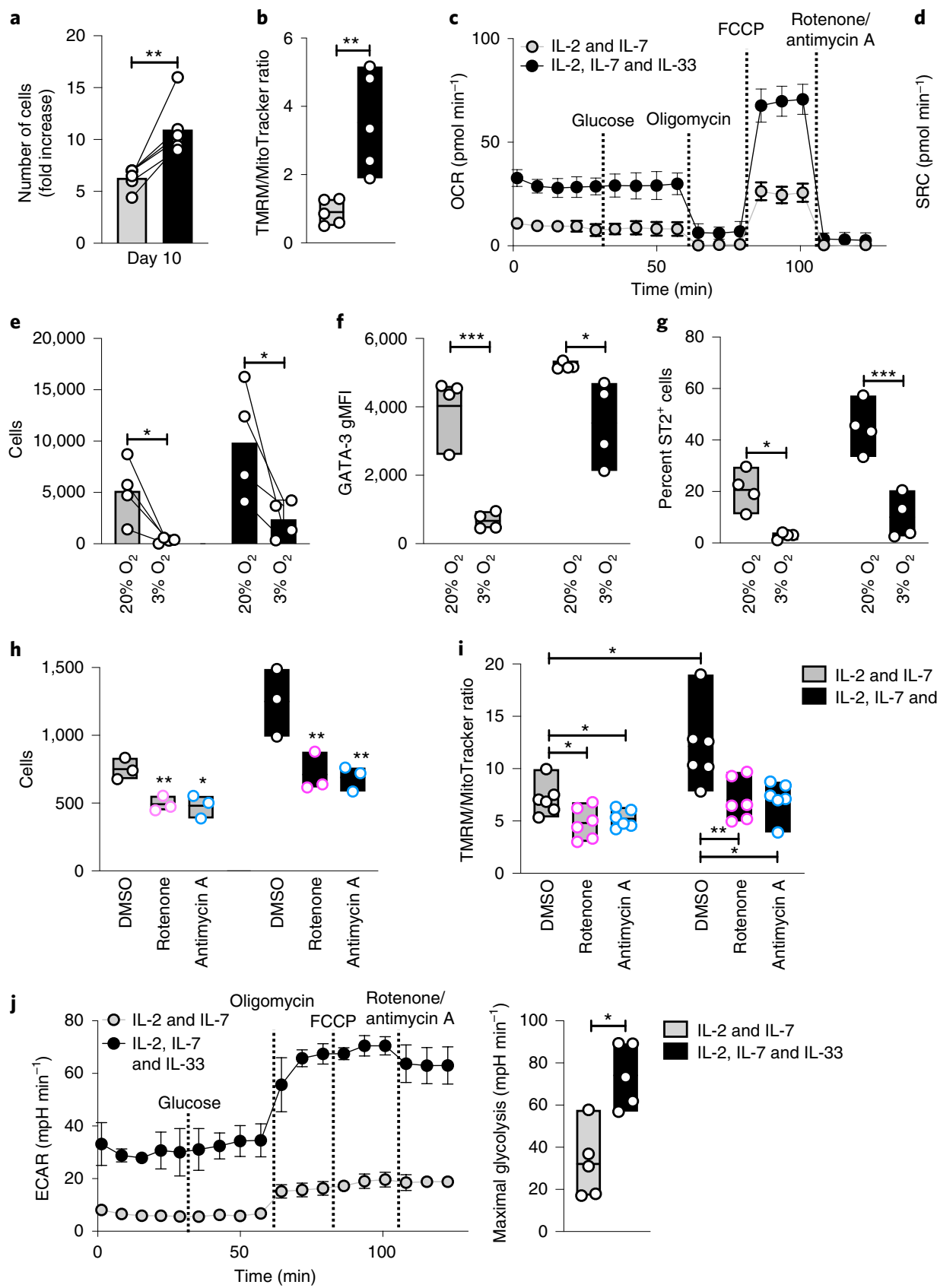
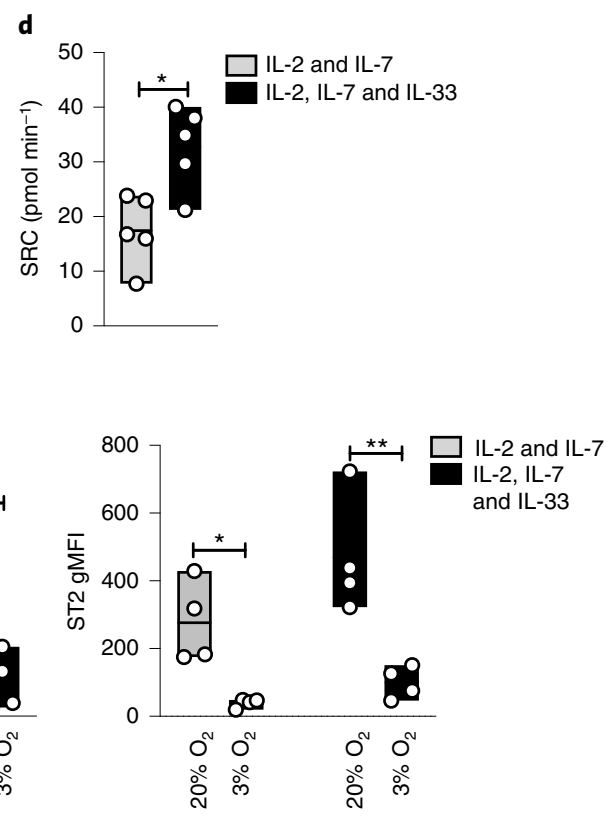

$\mathrm{IL}-2$ and IL-7

IL-2, IL-7 and IL-33

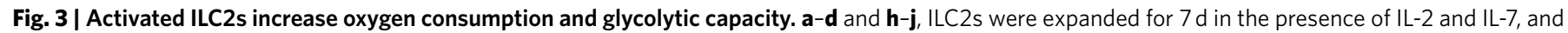

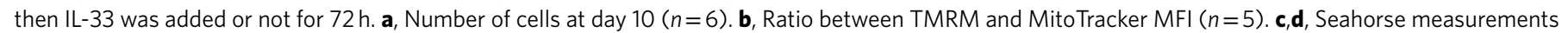
following the addition of glucose, oligomycin, fluoro-carbonyl cyanide phenylhydrazone (FCCP) and a combination of rotenone and antimycin A, including OCR (c) and SRC (maximal respiratory capacity after FCCP - basal respiratory capacity) (d) $(n=5)$. e-g, Cells were cultured at $20 \%$ or $3 \%$ oxygen in IL-2 and IL-7 or IL-2, IL-7 and IL-33 for 5 d. Cell count (e), GATA-3 expression (f), percent ST2+ cells and ST2 expression (g) were analyzed by FACS. h,i, Cells were treated for the last $18 \mathrm{~h}$ with $1 \mu \mathrm{M}$ rotenone, $1 \mu \mathrm{M}$ antimycin A or DMSO $(n=5)$. The total cell counts $(\mathbf{h})(n=3)$ and the ratio between TMRM and

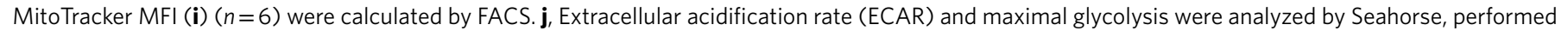
as in $\mathbf{c}$ and $\mathbf{d}(n=5)$. Data in $\mathbf{a}-\mathbf{d}$ and $\mathbf{j}$ are representative of five independent experiments with three to five donors each. Data in $\mathbf{e}-\mathbf{i}$ are representative of three independent experiments with at least three donors each. The bars in $\mathbf{a}, \mathbf{c}, \mathbf{e}$ and $\mathbf{j}$ (left plot) represent mean \pm s.e.m., and the floating bars in $\mathbf{b}, \mathbf{d}, \mathbf{f}-\mathbf{i}$

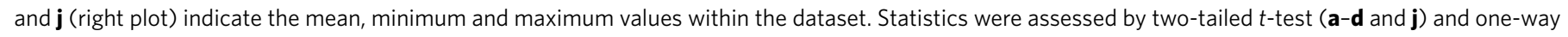
ANOVA with Tukey correction (e-i); NS, not significant $(P>0.05)$; ${ }^{\star} P<0.05 ;{ }^{\star \star} P<0.01 ;{ }^{\star \star \star} P<0.001$.

of ILC2 metabolomes after cytokine stimulation showed elevated BCAAs and glutamine and a loss of arginine compared to naive ILC2s (Fig. 4a and Supplementary Data 1). Specific changes that accompanied IL-33 exposure included an increase in pyruvate and lactate, consistent with augmented glycolysis (Fig. 3j). IL-33-activated ILC2s showed an increase in the expression of GLUT1, ASCT1 and
ASCT2 compared to naive levels (Fig. 4b), whereas CD98 levels were unchanged, suggesting that amino acid accumulation might not depend on increased uptake from the environment.

We next studied how these different nutrients are required for maintenance of mitochondrial activity in cytokine-activated ILC2s. We found that BCAAs are the main nutrient source maintaining 

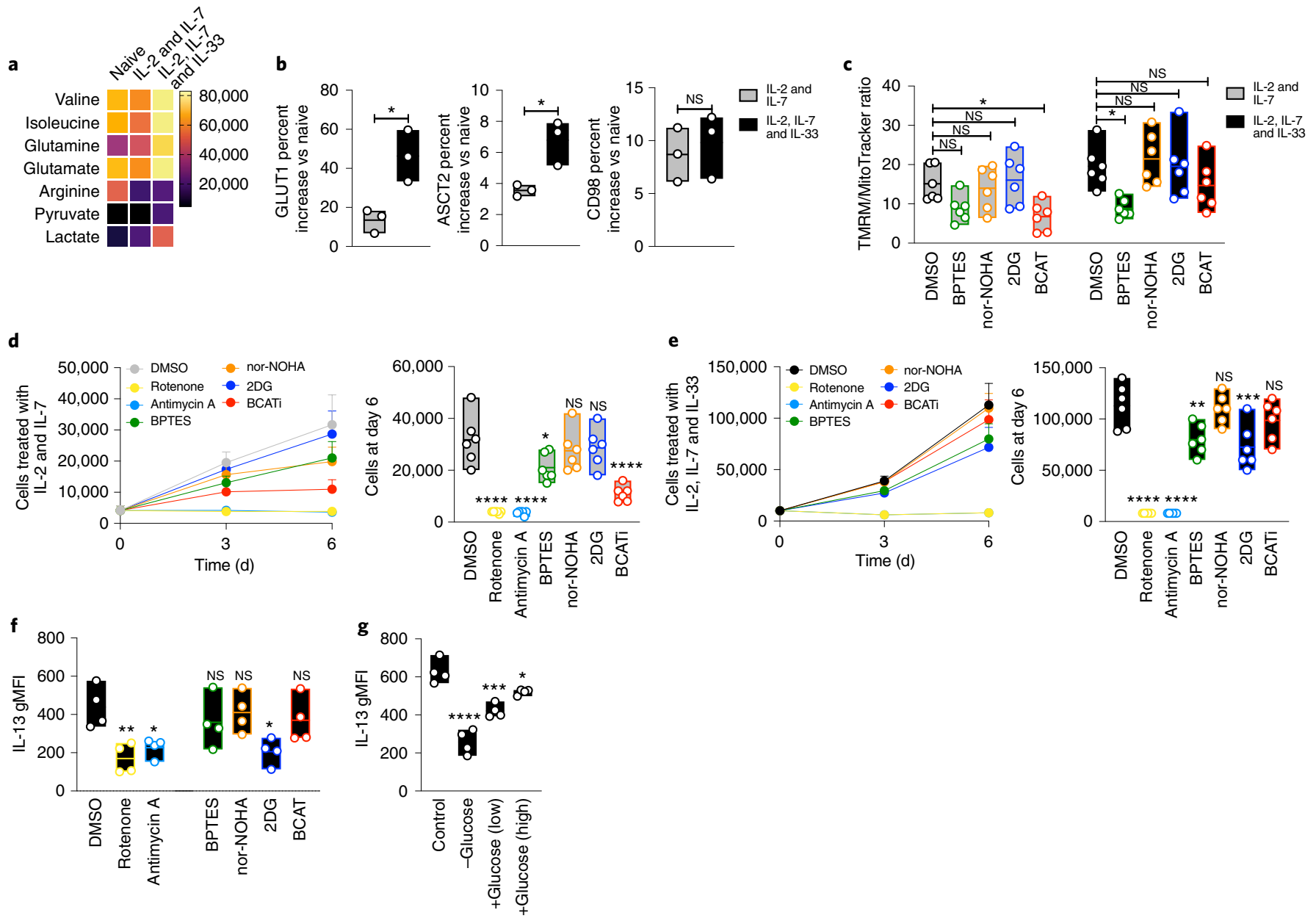

Fig. 4 | IL-33-stimulated ILC2s use amino acid metabolism to sustain cellular fitness and glucose for cytokine production. a-c, ILC2s were expanded for $7 \mathrm{~d}$ in IL-2 and IL-7, and IL-33 was added or not for $72 \mathrm{~h}$. $\mathbf{a}$, A heat map of amino acid intensities quantified by label-free mass spectrometry in naive cells and in ILC2s treated with either IL-2 and IL-7 or IL-2, IL-7 and IL-33 is shown. b, Percentage increase of nutrient receptors in primed (IL-2 and IL-7) or activated (IL-2, IL-7 and IL-33) cells versus in naive ILC2s $(n=3)$. c, ILC2s were cultured for $18 \mathrm{~h}$ with $1 \mu \mathrm{M}$ BPTES, $1 \mu \mathrm{M} 2 \mathrm{DG}, 1 \mu \mathrm{M}$ nor-NOHA, $1 \mu \mathrm{M}$ BCATi or DMSO in the indicated cytokine combinations. TMRM and MitoTracker were measured by FACS $(n=6)$. d,e, Cells were cultured for $6 \mathrm{~d}$ with IL-2 and IL-7 (d) or with IL-2, IL-7 and IL-33 (e) and $1 \mu \mathrm{M}$ rotenone, $1 \mu \mathrm{M}$ antimycin A, $1 \mu \mathrm{M}$ BPTES, $1 \mu \mathrm{M}$ 2DG, $1 \mu \mathrm{M}$ nor-NOHA, $1 \mu \mathrm{M}$ BCATi or DMSO. The cell counts at day 6 are shown $(n=6)$. f, Expression of IL-13. ILC2s were expanded in IL-2 and IL-7 for $7 \mathbf{d}$ with or without inhibitors as described in $\mathbf{d}$ and $\mathbf{e}$, and IL-33 was added for the last 6 h. $\mathbf{g}$, ILC2s were expanded in IL-2 and IL-7 for $7 \mathrm{~d}$, and IL-33 was added for the last $6 \mathrm{~h}$ to cells starved for $1 \mathrm{~h}$ and under a glucose add-back condition. IL-13 expression was monitored by FACS $(\mathbf{f}, \mathbf{g})(n=4)$. Data in a are summarized from four donors, and a minimum of two technical replicates were analyzed per run. Data in $\mathbf{b}$ are representative of three independent experiments with three donors each. Data in $\mathbf{c}$ are summarized from two experiments with three donors each and are representative of a total of four independent experiments. Data in $\mathbf{d}$ and $\mathbf{e}$ are summarized from two experiments with three donors each and are representative of a total of three independent experiments. Data in $\mathbf{f}$ and $\mathbf{g}$ are representative of a total of three independent experiments with at least three donors. Floating bars in $\mathbf{b}-\mathbf{g}$ indicate the mean, minimum and maximum values within the dataset. Bars in $\mathbf{d}$ and $\mathbf{e}$ (left plots) represent mean \pm s.e.m. Statistics were assessed by two-tailed $t$-test (b) and one-way ANOVA (c-g) with Dunnett correction; NS, not significant $(P>0.05) ;{ }^{\star} P<0.05 ;{ }^{* \star} P<0.01 ;{ }^{\star \star \star} P<0.001$.

OXPHOS in IL-2- and IL-7-stimulated ILC2s, while glutamine was also crucial in the context of IL-33 (Fig. 4c and Extended Data Fig. $8 \mathrm{a})$. By contrast, BCATi did not have any obvious effect (in line with previous findings ${ }^{48}$ ), and no changes in ILC2 mitochondrial mass or viability were detected under any conditions (Extended Data Fig. $8 \mathrm{~b}, \mathrm{c})$. We found an increase in the uptake of fluorescently labeled long-chain FA BODIPY only following IL-33 treatment (Extended Data Fig. 8d), consistent with a recent study showing the formation of FA lipid droplets following IL-33 chronic stimulation ${ }^{35}$. Still, inhibiting FA $\beta$-oxidation did not affect ILC2 mitochondrial mass or $\Delta \psi_{\mathrm{m}}$ (Extended Data Fig. 8e), suggesting that FAs might not play a crucial role in sustaining ILC2 bioenergetic needs. Additional studies are needed to fully clarify the role of FAs in human ILC2s. Curiously, inhibition of glycolysis (using 2DG) did not impact mito- chondrial polarization in cytokine-activated ILC2s, suggesting that these cells might couple glucose consumption to other functions.

To assess the effects of long-term inhibition of nutrient pathways, we extended the ILC2 cultures for $7 \mathrm{~d}$ (Fig. $4 \mathrm{~d}$,e and Extended Data Fig. 8f). As expected, cells did not survive in the presence of rotenone and antimycin A irrespective of cytokine combination. Long-term BPTES treatment impacted the survival of ILC2s when exposed to IL-2 and IL-7 or to IL-2, IL-7 and IL-33, while treatment with BCATi only had an effect in the absence of IL-33, consistent with our results on mitochondrial activity. In agreement with previous reports ${ }^{49-52}$ and our data on glycolytic capacity (Fig. 3j), long-term inhibition of glycolysis affected highly proliferative IL-33-stimulated ILC2s but not basal proliferation induced by IL-2 and IL-7 alone. These results indicate that amino acid 
metabolism remains a central orchestrator of cellular fitness during cytokine-induced ILC2 proliferation.

We next studied the effect of metabolic inhibitors on ILC2 cytokine production. Blocking glycolysis or ETC complexes I or III decreased IL-13 and IL-5 production (Fig. 4 f and Extended Data Fig. 9a,b). Amphiregulin production was less affected (Extended Data Fig. 9c), suggesting that pro- and anti-inflammatory ILC2 pathways might rely on different metabolic programs. Moreover, glucose starvation ablated IL-13 production, while glucose 'add-back' (at low or high concentration) partially restored IL-13 production (Fig. 4g and Extended Data Fig. 9d). It has been shown that glucose impacts lymphocyte function and proliferation through mTOR, an essential nutrient sensor ${ }^{35}$. Moreover, reactive oxygen species (ROS) have been reported to play a crucial role in mouse ILC activation ${ }^{53}$. We found that IL-33 induced ROS production in human ILC2s (Extended Data Fig. 9e). Treatment of ILC2s with a ROS scavenger or rapamycin (an mTOR inhibitor) reduced IL-13 production (Extended Data Fig. 9f). Taken together, these results demonstrate the unique role for glycolysis and ROS in coupling IL-33 activation to ILC2 cytokine production.

Here, we provide an in-depth characterization of human ILC2 metabolism at steady state and after cytokine activation. Our analysis identified the enhanced metabolic state of ILC2s compared to other innate lymphoid cell subsets and further deciphered the dichotomous nutrient pathways that sustain ILC2 survival, proliferation and function. Human ILC2 metabolism is poorly understood, with circulating ILC2s being described as resting naive cells with a migratory phenotype ${ }^{17,24}$. We show that they are instead highly energetic with a defined metabolic profile characterized by high expression of CD98 and elevated rates of amino acid uptake to sustain OXPHOS. These metabolic profiles provide cells with the capacity to respond to increased metabolic demands following activation ${ }^{28,31}$ and represent environment-specific metabolic adaptation ${ }^{54,55}$, suggesting that human circulating ILC2s might have metabolic 'experience. The heightened metabolic profile of circulating ILC2s was strongly OXPHOS/oxygen-dependent, as shown by the analysis of individuals with MDs and hypoxic experiments. Following activation, ILC2s do not undergo a metabolic switch from OXPHOS to glycolysis but rather maintain a dichotomous cellular metabolism with persistent OXPHOS and enhanced glycolysis. The former assures ILC2 survival (with glutamine as an anaplerotic substrate to maintain cell fitness), while glucose uptake and glycolysis sustain IL-13 production.

Our findings provide a working model for understanding how ILC2 metabolism conditions ILC function. Moreover, the peculiar metabolic features of steady-state and cytokine-activated ILC2s might represent potential targets for modulating these cells in diverse disease contexts.

\section{Online content}

Any methods, additional references, Nature Research reporting summaries, source data, extended data, supplementary information, acknowledgements, peer review information; details of author contributions and competing interests; and statements of data and code availability are available at https://doi.org/10.1038/ s41590-021-01043-8.

Received: 19 February 2021; Accepted: 9 September 2021; Published online: 22 October 2021

\section{References}

1. Eberl, G., Colonna, M., Di Santo, J. P. \& McKenzie, A. N. J. Innate lymphoid cells: a new paradigm in immunology. Science 348, aaa6566 (2015).

2. Spits, H. et al. Innate lymphoid cells-a proposal for uniform nomenclature. Nat. Rev. Immunol. 13, 145-149 (2013).

3. Vivier, E. et al. Innate lymphoid cells: 10 years on. Cell 174, 1054-1066 (2018).
4. Serafini, N. et al. Gata3 drives development of ROR $\gamma \mathrm{t}^{+}$group 3 innate lymphoid cells. J. Exp. Med. 211, 199-208 (2014).

5. Cupedo, T. et al. Human fetal lymphoid tissue-inducer cells are interleukin 17-producing precursors to $\mathrm{RORC}^{+} \mathrm{CD} 127^{+}$natural killer-like cells. Nat. Immunol. 10, 66-74 (2009).

6. Eberl, G. et al. An essential function for the nuclear receptor ROR $\gamma_{t}$ in the generation of fetal lymphoid tissue inducer cells. Nat. Immunol. 5, 64-73 (2004).

7. Artis, D. \& Spits, H. The biology of innate lymphoid cells. Nature 517, 293-301 (2015).

8. Bernink, J. H. et al. Human type 1 innate lymphoid cells accumulate in inflamed mucosal tissues. Nat. Immunol. 14, 221-229 (2013).

9. Lim, A. I. et al. Systemic human ILC precursors provide a substrate for tissue ILC differentiation. Cell 168, 1086-1100 (2017).

10. Robinette, M. L. et al. Transcriptional programs define molecular characteristics of innate lymphoid cell classes and subsets. Nat. Immunol. 16, 306-317 (2015).

11. Mjösberg, J. M. et al. Human IL-25- and IL-33-responsive type 2 innate lymphoid cells are defined by expression of CRTH2 and CD161. Nat. Immunol. 12, 1055-1062 (2011).

12. Neill, D. R. et al. Nuocytes represent a new innate effector leukocyte that mediates type-2 immunity. Nature 464, 1367-1370 (2010).

13. Hoyler, T. et al. The transcription factor GATA3 controls cell fate and maintenance of type 2 innate lymphoid cells. Immunity 37, 634-648 (2012)

14. Wilhelm, C. et al. An IL-9 fate reporter demonstrates the induction of an innate IL-9 response in lung inflammation. Nat. Immunol. 12, 1071-1077 (2011).

15. Mohapatra, A. et al. Group 2 innate lymphoid cells utilize the IRF4-IL-9 module to coordinate epithelial cell maintenance of lung homeostasis. Mucosal. Immunol. 9, 275-286 (2016).

16. Björklund, A. K. et al. The heterogeneity of human $\mathrm{CD} 127^{+}$innate lymphoid cells revealed by single-cell RNA sequencing. Nat. Immunol. 17, 451-460 (2016).

17. Mazzurana, L. et al. Tissue-specific transcriptional imprinting and heterogeneity in human innate lymphoid cells revealed by full-length single-cell RNA-sequencing. Cell Res. 31, 554-568 (2021).

18. Moro, $K$. et al. Innate production of $\mathrm{T}_{\mathrm{H}} 2$ cytokines by adipose tissue-associated c-Kit ${ }^{+} \mathrm{Sca}-1^{+}$lymphoid cells. Nature 463 , 540-544 (2010).

19. Stier, M. T. et al. IL-33 promotes the egress of group 2 innate lymphoid cells from the bone marrow. J. Exp. Med. 215, 263-281 (2018).

20. Huang, Y. et al. S1P-dependent interorgan trafficking of group 2 innate lymphoid cells supports host defense. Science 359, 114-119 (2018).

21. Miller, M. M. et al. BATF acts as an essential regulator of IL-25responsive migratory ILC2 cell fate and function. Sci. Immunol. 5, eaay3994 (2020).

22. Ricardo-Gonzalez, R. R. et al. Tissue-specific pathways extrude activated ILC2s to disseminate type 2 immunity. J. Exp. Med. 217, e20191172 (2020).

23. Huang, Y. et al. IL-25-responsive, lineage-negative KLRG ${ }^{\text {hi }}$ cells are multipotential 'inflammatory' type 2 innate lymphoid cells. Nat. Immunol. 16, 161-169 (2015)

24. van der Ploeg, E. K. Steroid-resistant human inflammatory ILC2s are marked by CD45RO and elevated in type 2 respiratory diseases. Sci. Immunol. 6, eabd3489 (2021).

25. Klose, C. S. N. \& Artis, D. Innate lymphoid cells control signalling circuits to regulate tissue-specific immunity. Cell Res. 30, 475-491 (2020).

26. Weinberg, S. E., Sena, L. A. \& Chandel, N. S. Mitochondria in the regulation of innate and adaptive immunity. Immunity 42, 406-417 (2015).

27. Buck, M. D. et al. Mitochondrial dynamics controls T cell fate through metabolic programming. Cell 166, 63-76 (2016).

28. Buck, M. D., O'Sullivan, D. \& Pearce, E. L. T cell metabolism drives immunity. J. Exp. Med. 212, 1345-1360 (2015).

29. van der Windt, G. J. W., Chang, C.-H. \& Pearce, E. L. Measuring bioenergetics in $\mathrm{T}$ cells using a Seahorse extracellular flux analyzer. Curr. Protoc. Immunol. 113, 3.16B.1-3.16B.14 (2016).

30. Hartmann, F. J. et al. Single-cell metabolic profiling of human cytotoxic T cells. Nat. Biotechnol. 39, 186-197 (2021).

31. Puleston, D. J., Villa, M. \& Pearce, E. L. Ancillary activity: beyond core metabolism in immune cells. Cell Metab. 26, 131-141 (2017).

32. Ma, E. H. et al. Metabolic profiling using stable isotope tracing reveals distinct patterns of glucose utilization by physiologically activated CD8 ${ }^{+}$ T cells. Immunity 51, 856-870 (2019).

33. Nicoli, F. et al. Naive CD8 ${ }^{+}$T-cells engage a versatile metabolic program upon activation in humans and differ energetically from memory $\mathrm{CD}^{+} \mathrm{T}$-cells. Front. Immunol. 9, 2736 (2018).

34. O'Sullivan, D. et al. Memory $\mathrm{CD}^{+} \mathrm{T}$ cells use cell intrinsic lipolysis to support the metabolic programming necessary for development. Immunity 41, 75-88 (2014). 
35. Pearce, E. L. et al. Enhancing CD8 T cell memory by modulating fatty acid metabolism. Nature 460, 103-107 (2009).

36. Wilhelm, C. et al. Critical role of fatty acid metabolism in ILC2-mediated barrier protection during malnutrition and helminth infection. J. Exp. Med. 213, 1409-1418 (2016)

37. Karagiannis, F. et al. Lipid-droplet formation drives pathogenic group 2 innate lymphoid cells in airway inflammation. Immunity 52, 620-634 (2020).

38. Monticelli, L. A. et al. Arginase 1 is an innate lymphoid cell-intrinsic metabolic checkpoint controlling type 2 inflammation. Nat. Immunol. 17, 656-665 (2016).

39. Surace, L. et al. Polarized mitochondria as guardians of NK cell fitness. Blood Adv. 5, 26-38 (2020)

40. Rambold, A. S. \& Pearce, E. L. Mitochondrial dynamics at the interface of immune cell metabolism and function. Trends Immunol. 39, 6-18 (2018).

41. Geiger, R. et al. L-Arginine modulates $\mathrm{T}$ cell metabolism and enhances survival and anti-tumor activity. Cell 167, 829-842 (2016).

42. Carr, E. L. et al. Glutamine uptake and metabolism are co-ordinately regulated by ERK/MAPK during T lymphocyte activation. J. Immunol. 185, 1037-1044 (2010).

43. Wolfson, R. L. \& Sabatini, D. M. The dawn of the age of amino acid sensors for the mTORC1 Pathway. Cell Metab. 26, 301-309 (2017).

44. Kelly, B. \& Pearce, E. L. Amino assets: how amino acids support immunity. Cell Metab. 32, 154-175 (2020).

45. Lim, A. I. et al. IL-12 drives functional plasticity of human group 2 innate lymphoid cells. J. Exp. Med. 213, 569-583 (2016).

46. Bartemes, K. R., Kephart, G. M., Fox, S. J. \& Kita, H. Enhanced innate type 2 immune response in peripheral blood from patients with asthma. J. Allergy Clin. Immunol. 134, 671-678 (2014).

47. Li, Q. et al. E3 ligase VHL promotes group 2 innate lymphoid cell maturation and function via glycolysis inhibition and induction of interleukin-33 receptor. Immunity 48, 258-270 (2018)

48. Shao, D. et al. Glucose promotes cell growth by suppressing branched-chain amino acid degradation. Nat. Commun. 9, 2935 (2018).
49. Jacobs, S. R. et al. Glucose uptake is limiting in T cell activation and requires CD28-mediated Akt-dependent and independent pathways. J. Immunol. 180, 4476-4486 (2008).

50. Sukumar, M. et al. Inhibiting glycolytic metabolism enhances CD8 ${ }^{+} \mathrm{T}$ cell memory and antitumor function. J. Clin. Invest. 123, 4479-4488 (2013).

51. Tanimine, N. et al. Differential effects of 2-deoxy-D-glucose on in vitro expanded human regulatory T cell subsets. PLoS ONE 14, e0217761 (2019).

52. Menk, A. V. et al. Early TCR signaling induces rapid aerobic glycolysis enabling distinct acute $\mathrm{T}$ cell effector functions. Cell Rep. 22, 1509-1521 (2018).

53. Di Luccia, B. et al. ILC3s integrate glycolysis and mitochondrial production of reactive oxygen species to fulfill activation demands. J. Exp. Med. 216, 2231-2241 (2019)

54. Pearce, E. L. \& Pearce, E. J. Metabolic pathways in immune cell activation and quiescence. Immunity 38, 633-643 (2013).

55. Pearce, E. L., Poffenberger, M. C., Chang, C.-H. \& Jones, R. G. Fueling immunity: insights into metabolism and lymphocyte function. Science 342, 1242454 (2013).

Publisher's note Springer Nature remains neutral with regard to jurisdictional claims in published maps and institutional affiliations.

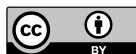

Open Access This article is licensed under a Creative Commons Attribution 4.0 International License, which permits use, sharing, adaptation, distribution and reproduction in any medium or format, as long as you give appropriate credit to the original author(s) and the source, provide a link to the Creative Commons license, and indicate if changes were made. The images or other third party material in this article are included in the article's Creative Commons license, unless indicated otherwise in a credit line to the material. If material is not included in the article's Creative Commons license and your intended use is not permitted by statutory regulation or exceeds the permitted use, you will need to obtain permission directly from the copyright holder. To view a copy of this license, visit http://creativecommons. org/licenses/by/4.0/.

(C) The Author(s) 2021 


\section{Methods}

Cell isolation from blood and tissue samples. Blood samples from healthy donors were randomly selected (age and sex) and obtained from Establissement Francais du Sang (EFS) under protocol HS 2105-24405. Peripheral blood mononuclear cells (PBMCs) from individuals with MD were obtained from the Unit of Neurology and Neuromuscular Disorders at 'University of Messina' with informed consent through an Institutional Review Board protocol (protocol 88/17 del 31 sett 2017) in compliance with ethical regulations. No statistical methods were used to predetermine sample sizes, but our sample sizes are similar to those reported in previous publications ${ }^{9,11,17}$. The number of participants for each experiment was dependent on donors' availability. No direct compensation was given by the authors to the healthy donors or individuals with MD. Isolation of human PBMCs and single-cell suspensions was achieved by Ficoll-Paque (GE Healthcare) density gradient centrifugation. Data collection and analysis were not blinded to the conditions of the experiments.

Cell culture and reagents. All in vitro culture experiments were performed in Yssel's medium prepared in house by using IMDM (Invitrogen) and $0.25 \%$ (wt/ vol) bovine serum albumin (Sigma), $1.8 \mathrm{mg} \mathrm{liter}^{-1} 2$-amino ethanol, $40 \mathrm{mg} \mathrm{liter}^{-1}$ apo-transferrin, $5 \mathrm{mg} \mathrm{liter}^{-1}$ insulin and penicillin/streptomycin and supplemented with $2 \%$ human $A B$ serum (EFS). FACS-sorted cells were plated in the presence of the human cytokines IL-2 (50 $\mathrm{ng} \mathrm{ml}^{-1}$; Miltenyi), IL-7 (50 ng ml-1; Miltenyi) and IL-33 (50 $\left.\mathrm{ng} \mathrm{ml}^{-1} ; \mathrm{R} \& \mathrm{D}\right)$, which were provided in various combinations and at specific times as indicated. For the hypoxia experiments, cells were cultured at 3\% oxygen in the XVivo System (BioSperix) at constant pressure, humidity and $5 \% \mathrm{CO}_{2}$. Chemical inhibitors used to study metabolic pathways (2DG, BPTES, etomoxir, TTFA, antimycin A, rotenone, oligomycin A, MitoTempo and rapamycin) were purchased from Sigma. BCATi and nor-NOHA were purchased from Cayman Chemicals. Cell viability was assessed by Annexin- $\mathrm{V}$ and live-dead staining by FACS. Cells were counted at the microscope after trypan blue staining or, when in limited number, by FACS.

Flow cytometry and cell sorting. Cells were stained with surface antibodies and Fixable Viability Dye eFluor 506 (eBioscience) in PBS supplemented with $2 \%$ fetal calf serum for $30 \mathrm{~min}$ on ice. For experiments involving intracellular staining of cytokines, cells were stimulated for $6 \mathrm{~h}$ with cytokines, and, during the last $3 \mathrm{~h}$, Golgi Plug and Golgi Stop (BD) were added to the cultures. Cells were washed with PBS and fixed and permeabilized for $45 \mathrm{~min}$ at room temperature using a Cytofix/ Cytoperm kit (BD). Intracellular staining was performed at room temperature for $30 \mathrm{~min}$ in the dark. Annexin- $\mathrm{V}$ staining was performed using Annexin-V-binding buffer (BD). Samples were acquired with an LSRFortessa (BD) and analyzed by FlowJ10.7.1 (TreeStar). For cell sorting, PBMCs were depleted of T cells, B cells, plasmacytoid dendritic cells, monocytes and erythrocytes by labeling with biotin-conjugated antibodies followed by anti-biotin microbeads and AutoMACS separation (Miltenyi) according to manufacturer's instruction. Cells were sorted in bulk to a purity of $\geq 99 \%$ (FACSAria II; BD).

Antibodies. Surface GLUT1 expression was monitored as a function of binding to its ligand, the envelope glycoprotein of the human $\mathrm{T}$ lymphotropic virus (HTLV). A recombinant HTLV envelope receptor-binding domain $\left(\mathrm{H}_{\mathrm{RBD}}\right)$ fused to an enhanced green fluorescent protein (eGFP)-coding sequence was used as previously described ${ }^{56}$. Surface ASCT2 was similarly evaluated; expression was monitored as a function of binding to its ligand, the RD114 envelope glycoprotein of the feline endogenous retrovirus, fused with a murine $\mathrm{Fc}$ tag and revealed with an Alexa Flour-647-conjugated anti-mouse IgG (Invitrogen).

Antibodies for ILC2 enrichment, including anti-hCD3 biotin (clone OKT3, 13-0037-82), anti-hCD4 biotin (RPA-T4, 13-0049-82), anti-hCD19 biotin (HIB19, 13-0199-82), anti-hCD14 biotin (61D3, 13-0149-82), anti-hCD123 biotin (6H6, 13-1239-82) and anti-hCD235a biotin (HIR2 GA-R2, 13-998782 ), were purchased from eBioscience. Antibodies used for ILC2 sorting, including anti-hCD3 FITC (UCHT1, 11-0038), anti-hCD4 FITC (OKT4, 11-0048), anti-hCD5 FITC (UCHT2, 11-0059), anti-h $\alpha \beta$ TCR FITC (IP26, 564451), anti-h $\gamma \delta$ TCR FITC (B1.1, 11-9986) and anti-hCD127 (IL-7R $\alpha)$ PE-Cy7 (eBioRDR5, 25-1278-42) were purchased from eBioscience. Anti-hCD14 FITC (TUK4, 130-080-701), anti-hCD19 FITC (LT19, 130-104-650) and anti-hCD159a (NKG2A) PE (REA110, 130-113-566) were purchased from Miltenyi. Anti-hCD294 (CRTH2) Alexa Fluor 647 (BM16, 558042) and anti-hCD7 BV711 (M-T701, 564018) were purchased from BD. Anti-hCD45 AF700 (HI30, 560566), anti-hCD94 APC-Fire750 (DX22, 305518), anti-hCD117 BV605 (104D2, 313218), anti-hCD16 BV650 (3G8, 302042) and anti-hCD56 BV756 $(5.1 \mathrm{H} 11,362550)$ were purchased from BioLegend. Antibodies for FACS analysis (extracellular and intracellular staining), including anti-hAnnexinV BV395 (564871), anti-hCD3 BUV737 (UCHT1, 612750), anti-hCD5 BUV737 (UCHT2, 612842), anti-hCD14 BUV737 (M5E2, 612763), anti-hCD19 BUV737 (SJ25C1, 612756), anti-hCD45 BV805 (HI30, 612891) and anti-hIL-13 BV421 (JES10-5A2, 624124), were purchased from BD. Anti-hST2 APC (hIL33Rcap, 17-9338-42) and anti-hAmphiregulin (AREG559, 17-5370-42) were purchased from eBioscience, and anti-hIL-5 (TRFK5, 504311) and anti-hHIF1 $\alpha$ (546-16, 359704) were purchased from BioLegend.
Mitochondrial mass, membrane potential by FACS and confocal microscopy and FA uptake. Mitochondrial mass, membrane potential and FA uptake of freshly sorted or cytokine-activated ILC2s were assessed by staining cells with $50 \mathrm{nM}$ MitoTracker Green FM (Thermo Fisher), $25 \mathrm{nM}$ TMRM (Sigma-Aldrich) and BODIPY FL- $\mathrm{C}_{16}$ (Thermo Fisher), respectively, for $30 \mathrm{~min}$ at $37^{\circ} \mathrm{C}$ and $5 \%$ $\mathrm{CO}_{2}$. Cells were washed twice in cold $1 \times$ PBS, stained with surface antibodies and analyzed by FACS. For confocal microscopy, cells were stained at $37^{\circ} \mathrm{C}$ for 30 min with $300 \mathrm{ng} \mathrm{ml}^{-1}$ of Hoechst H33342 (Life Technologies) to stain nuclei, $100 \mathrm{nM}$ MitoTracker Green FM to stain mitochondria and $25 \mathrm{nM}$ TMRM to asses mitochondrial membrane potential (non-quenching mode, TMRM maintained in the cell medium). Cells were plated in a 384 -well plate (40,000 cells per well), and image acquisitions of multiple fields per well were performed on an automated confocal microscope (OPERA QEHS, Perkin Elmer) using $\times 60$ objectives, excitation lasers at 405,488 and $561 \mathrm{~nm}$ and emission filters at 450, 540 and $600 \mathrm{~nm}$, respectively. Confocal images were transferred to the Columbus Image Data Storage and Analysis System (Perkin Elmer) for high content analyses as previously reported ${ }^{57}$ and used the standard deviation/mean approach ${ }^{58}$.

Metabolite extraction, mass spectrometry and data analysis. Methods for metabolite extraction, data acquisition and data analysis were developed and performed by General Metabolics. FACS-sorted ILC2s (100,000 cells) were pelleted and washed in prewarmed $\left(37^{\circ} \mathrm{C}\right)$ ammonium carbonate $(75 \mathrm{mM})$ washing buffer. Preheated $\left(70^{\circ} \mathrm{C}\right) 70 \%$ ethanol $(99.9 \%$ purity) extraction solvent was added, and samples were incubated for $3 \mathrm{~min}$. After centrifugation $\left(8,000 \mathrm{~g}\right.$ for $10 \mathrm{~min}$ at $\left.0^{\circ} \mathrm{C}\right)$, the supernatants were collected and stored at $-80^{\circ} \mathrm{C}$ until analysis. The analysis was performed on a platform consisting of an Agilent Series 1100 LC pump coupled to a Gerstel MPS2 autosampler and an Agilent 6520 Series Quadrupole time-of-flight mass spectrometer equipped with an electrospray source operated in negative and positive mode as previously reported ${ }^{59}$. All steps of data processing and analysis were performed with Matlab R2010b (MathWorks) using functions embedded in the bioinformatics, statistics, database and parallel computing toolboxes. For each run, a matrix list was produced with the intensity of each mass peak in each analyzed sample. An accurate $\mathrm{m} / \mathrm{z}$ was recalculated with a weighted average of the values, and a list of putative metabolites was compiled from the KEGG database. Raw intensities for each metabolite are included in Supplementary Data 1. For each ion, the best metabolite match was chosen among all candidates based on the deviation from the theoretical $\mathrm{m} / \mathrm{z}$ and a heuristic probability associated with the theoretical ion fragment, which was set lower for, for example, rare adducts, fragments or molecules containing several heteroatoms. Significant changes in metabolite levels compared to the entire dataset were determined by calculating $z$ scores for each sample and ion individually, as previously reported ${ }^{56}$. Results were run in iPath3 (https://pathways.embl.de), an online tool for data mapping. A Principal-component analysis was applied to the samples coming from two different screenings using the stats $\mathrm{R}$ package. The screenings were simultaneously analyzed considering only metabolites present in both screenings (total $=122$ metabolites). The first principal component indicated that most of the variance of the data corresponded to the screening effect, as expected from the batch effect that each screening represented. The second most variable effect corresponded to the donors. Pathway analysis was done with Qlucore Omic Explorer v3.

RNA isolation, library construction, sequencing and analysis. ILC2s $\left(10^{3}\right.$ cells were sorted by FACS directly into $50 \mathrm{ml}$ of lysis/binding buffer (Life Technologies). mRNA was captured with $15 \mathrm{ml}$ of Dynabeads oligo(dT) (Life Technologies), washed according to manufacturer's instructions and eluted at $85^{\circ} \mathrm{C}$ with $6 \mathrm{ml}$ of $10 \mathrm{mM}$ Tris-HCl (pH 7.5). We used a derivation of MARS-seq as described in ref. ${ }^{60}$, developed for single-cell RNA-seq, to produce expression libraries of two replicates per population. Libraries were sequenced at an average depth of 5 million reads per library on an Ilumina NextSeq and aligned to the human reference genome (hg19). Reads were mapped using hisat (version 0.1.6); duplicate reads were filtered if they had identical unique molecular identifiers. Expression levels were calculated and normalized to the total number of reads using HOMER software. RNA-seq datasets have been deposited in the Gene Expression Omnibus (GEO) public repository (accession number GSE183669).

Transcriptional profiling by BioMark. ILC2s were sorted as small bulks ( 25 cells)

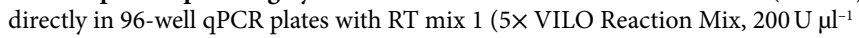
SUPERase-In, 10\% NP-40 and nuclease free water). Reverse transcription was performed according to manufacturer's protocols (Fluidigm). The dynamic Array IFC chip was prepared according to manufacturer's protocols and analyzed in the Biomark System (Fluidigm). Cycle threshold $\left(C_{\mathrm{t}}\right)$ values were collected from the machine and processed using Prism 8.

Cellular metabolism by Seahorse extracellular flux analyzer. OCRs and ECARs were measured for freshly sorted, IL-2-, IL-7- and/or IL-33-stimulated ILC2s $(100,000$ cells). XF medium (non-buffered RPMI 1640 containing $10 \mathrm{mM}$ glucose, $2 \mathrm{mM} \mathrm{L}$-glutamine and $1 \mathrm{mM}$ sodium pyruvate) was used under basal conditions. Addition of $1 \mu \mathrm{M}$ oligomycin, $1.5 \mu \mathrm{M}$ FCCP and $100 \mathrm{nM}$ rotenone $+1 \mu \mathrm{M}$ 
antimycin A was performed using portal injection in a 96-well XF or XFe Extracellular Flux Analyzer (Seahorse Bioscience)

Statistical analysis. Flow cytometry data were analyzed using FlowJo v.10 (TreeStar). Statistical analyses were done using a two-tailed $t$-test or one-way ANOVA test with Dunnett correction when comparing multiple groups to specific conditions or a Tukey correction for multiple comparisons when comparing more than two conditions of interest (GraphPad Prism v.8 and v.9). The statistical tests, replicate experiments and $P$ values are all cited in the figures and/or figure captions. Statistical tests were justified as appropriate for every figure, and the data meet the assumptions of the tests. The ranges of $x$ and $y$ axes for scatter plots were determined to include all of the data points. The sample size for each experiment and the replicate number of experiments are included in the figure legends as well as the specific test used for the analysis.

Reporting Summary. Further information on research design is available in the Nature Research Reporting Summary linked to this article.

\section{Data availability}

All the data generated and/or analyzed during this study are included in this manuscript as Supplementary Information. RNA-seq datasets have been deposited in the GEO public repository (accession number GSE183669). Source data are provided with this paper

\section{References}

56. Manel, N. et al. The ubiquitous glucose transporter GLUT-1 is a receptor for HTLV. Cell 115, 449-459 (2003).

57. Escoll, P. et al. Legionella pneumophila modulates mitochondrial dynamics to trigger metabolic repurposing of infected macrophages. Cell Host Microbe 22 302-316 (2017).

58. Duchen, M. R., Surin, A. \& Jacobson, J. Imaging mitochondrial function in intact cells. Methods Enzymol. 361, 353-389 (2003).

59. Fuhrer, T. et al. High-throughput, accurate mass metabolome profiling of cellular extracts by flow injection-time-of-flight mass spectrometry. Anal. Chem. 83, 7074-7080 (2011).

60. Jaitin, D. A. et al. Massively parallel single-cell RNA-seq for markerfree decomposition of tissues into cell types. Science 343, 776-779 (2014)

\section{Acknowledgements}

We thank all the members of the Innate Immunity Unit for helpful discussions, the Centre de Recherche Translationnelle and the logistic department of Institut Pasteur. We thank the CB-UTechS platform for cytometry support and the Imagopole-CiTech (part of France-BioImaging supported by ANR grant no. ANR-10-INSB-04-01,

Conseil de la Region Ile-de-France, FRM) for technical support. The Innate Immunity Unit is supported by grants from the Institut National de la Santé et de la Recherche Médicale (INSERM), Institut Pasteur, the Agence National pour le Recherche (ANR) and the European Research Council (ERC) under the European Union's Horizon 2020 research and innovation program (695467, ILC_REACTIVITY). A.T. is supported by European Union's Horizon 2020 research and innovation program under the Marie Skłodowska-Curie grant agreement no. 765104. C.B.s group was supported by the Fondation pour la Recherche Médicale (FRM) grant no. EQU201903007847 and the grant no. ANR-10-LABX-62-IBEID. L.S. was supported by an SNSF-Early PostDoc. Mobility fellowship and a Marie Curie grant (H2020- MSCA-IF-2017).

\section{Author contributions}

The study was conceptualized by L.S. and J.P.D. Experiments were coordinated by L.S. FACS and subsequent analyses were performed by L.S., C.A.C., A.T., J.-M.D. and S.M. Confocal microscopy analysis was performed by P.E., C.B. and L.S. Bioinformatic analyses were performed by N.P. and V.G. RNA-seq experiments were conducted by I.A. O.M., V.D., N.T., D.T. and A.C. provided resources. L.S. and J.P.D. wrote the paper. Funding was acquired by L.S. and J.P.D., and the study was supervised by J.P.D.

\section{Competing interests}

The authors declare no competing interests.

\section{Additional information}

Extended data is available for this paper at https://doi.org/10.1038/s41590-021-01043-8.

Supplementary information The online version contains supplementary material available at https://doi.org/10.1038/s41590-021-01043-8.

Correspondence and requests for materials should be addressed to James P. Di Santo.

Peer review information Nature Immunology thanks Jörg Fritz and the other, anonymous, reviewer(s) for their contribution to the peer review of this work. L. A. Dempsey was the primary editor on this article and managed its editorial process and peer review in collaboration with the rest of the editorial team.

Reprints and permissions information is available at www.nature.com/reprints. 
a

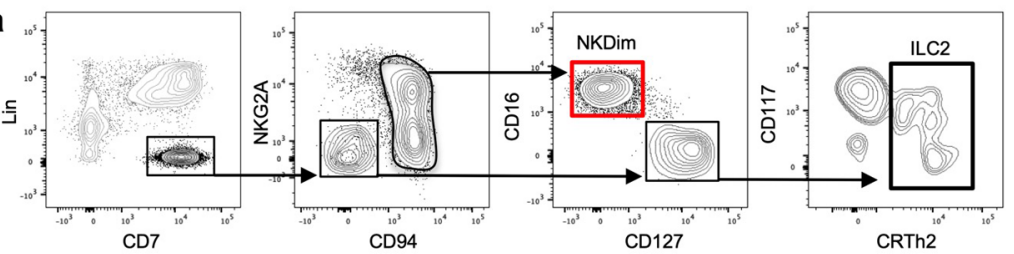

C

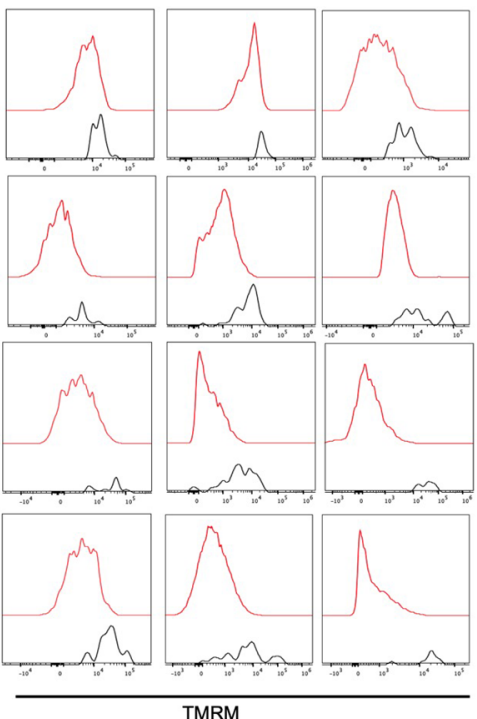

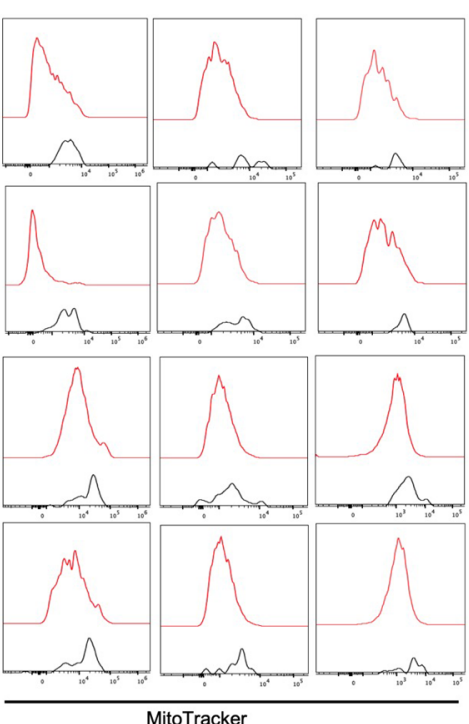

MitoTracker

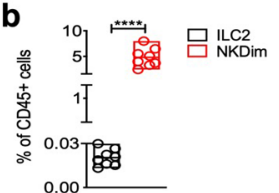

0.00

Extended Data Fig. 1 | Human ILC2 have fused and polarized mitochondria. a, ILC2 Gating strategy Live CD45+ CD7+ Lin $^{-}$CD94- NKG2A- CD56- CD16$\mathrm{CD}_{127}{ }^{+} \mathrm{CRTh}_{2}{ }^{+}$(ILC2) and Live CD45+ CD7 ${ }^{+} \mathrm{Lin}^{-} \mathrm{CD} 94^{+} \mathrm{CD} 16^{+}$(NKDim). b, Percentage of ILC2 and NKDim in live CD45+ cells. c, FACS measurement of TMRM and MitoTracker in healthy donors of fresh ILC2 and NKDim $(n=12)$. d, Confocal microscopy in fresh ILC2 and NKDim $(n=7)$. e, MitoTracker and TMRM intensity (SD/Mean). f, ATP and ADP intensities quantified by label-free mass spectrometry in freshly sorted ILC2 and NKDim cells $(n=3)$. a-b, Data representative of 10 independent experiments with 2 to 4 donors each. $\mathbf{c}$, Data from 12 healthy donors from at least 5 independent experiments. $\mathbf{d}$-e, Data representative of 2 independent experiments with at least 3 donors. Dots are single cells in the analyzed field. Scale bar $5 \mu \mathrm{m}$. $\mathbf{f}$, Each dot represents a donor. A minimum of 2 technical replicates were analyzed per run. $\mathbf{b}, \mathbf{e}, \mathbf{f}$, Floating bars indicate the mean, minimum and maximum values within the dataset. Statistics were assessed by two tailed t-test (b,e,f), not significant (ns), p $>0.05 ;{ }^{\star \star} p<0.01 ;{ }^{\star \star \star *} p<0.0001$. 
a

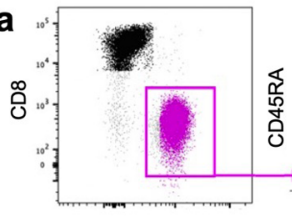

CD4

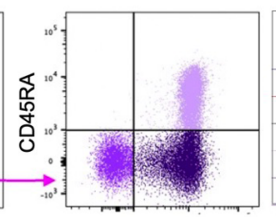

CD27

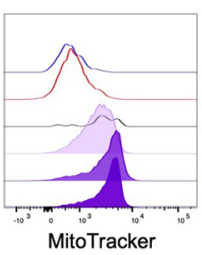

MitoTracker

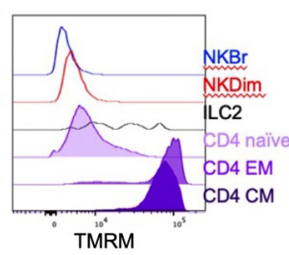

TMRM
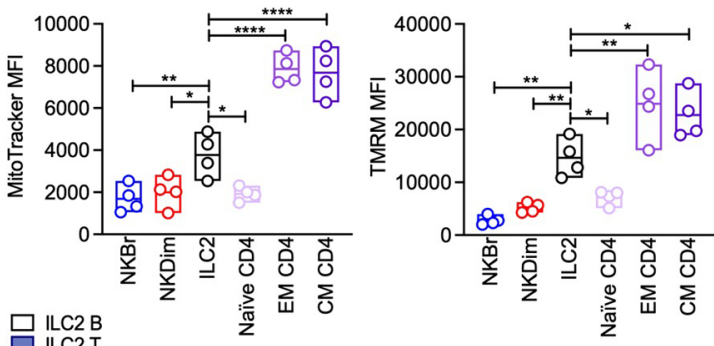

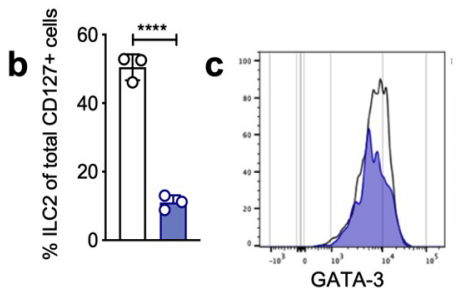

e

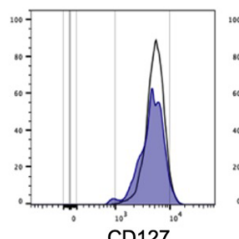

CD127

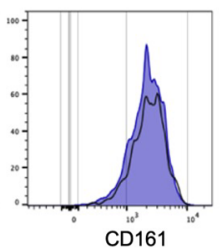

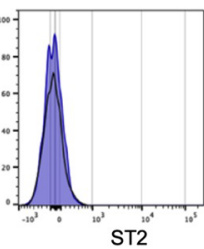
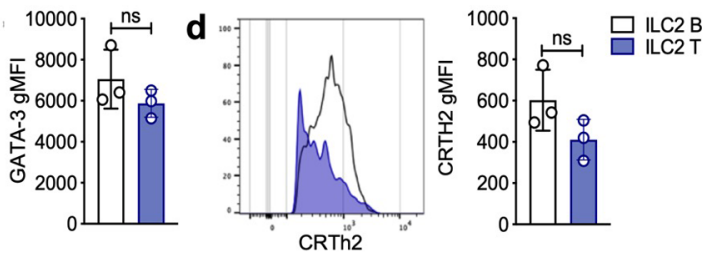

f
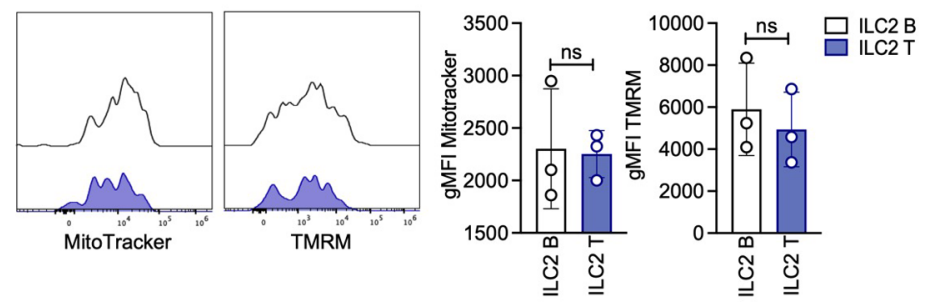

Extended Data Fig. 2 | ILC2 have enhanced mitochondrial activity compared to naïve T cells. a, Gating strategy for CD4 ${ }^{+}$T cells subsets in the blood of healthy donors. FACS measurement of TMRM and MitoTracker gMFI in the indicated subsets $(n=4)$. b-f, Paired blood and tonsil samples were collected from pediatric donors. The percentage of ILC2 (b), GATA-3 gMFI (c), CRTH2 gMFI (d), representative plots of CD127, CD161, ST2 (e), TMRM and Mitotracker gMFI (f) were monitored by FACS $(n=3)$. $\mathbf{a}$, Data representative of 2 experiments with at least 3 donors each. $\mathbf{b}-\mathbf{f}$, Data are representative of 2 independent experiments with 3 donors each. a, Floating bars indicate the mean, minimum and maximum values within the dataset. b-f, Bars are \pm s.e.m. Statistics were assessed by one-way ANOVA with Dunnett correction (a) and two tailed t-test (b-f), not significant (ns), p >0.05; ${ }^{\star}$ < < 0.05; ${ }^{\star \star} \mathrm{p}<0.01 ;{ }^{\star \star \star \star} \mathrm{p}<0.0001$. 
a

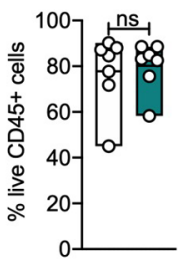

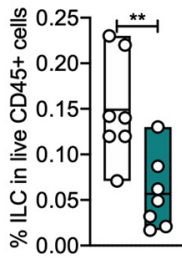

b
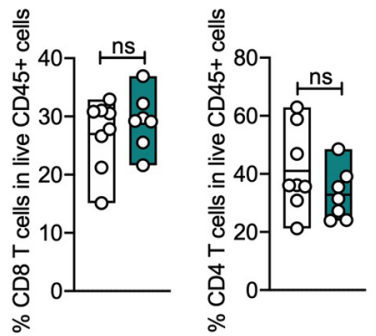

C
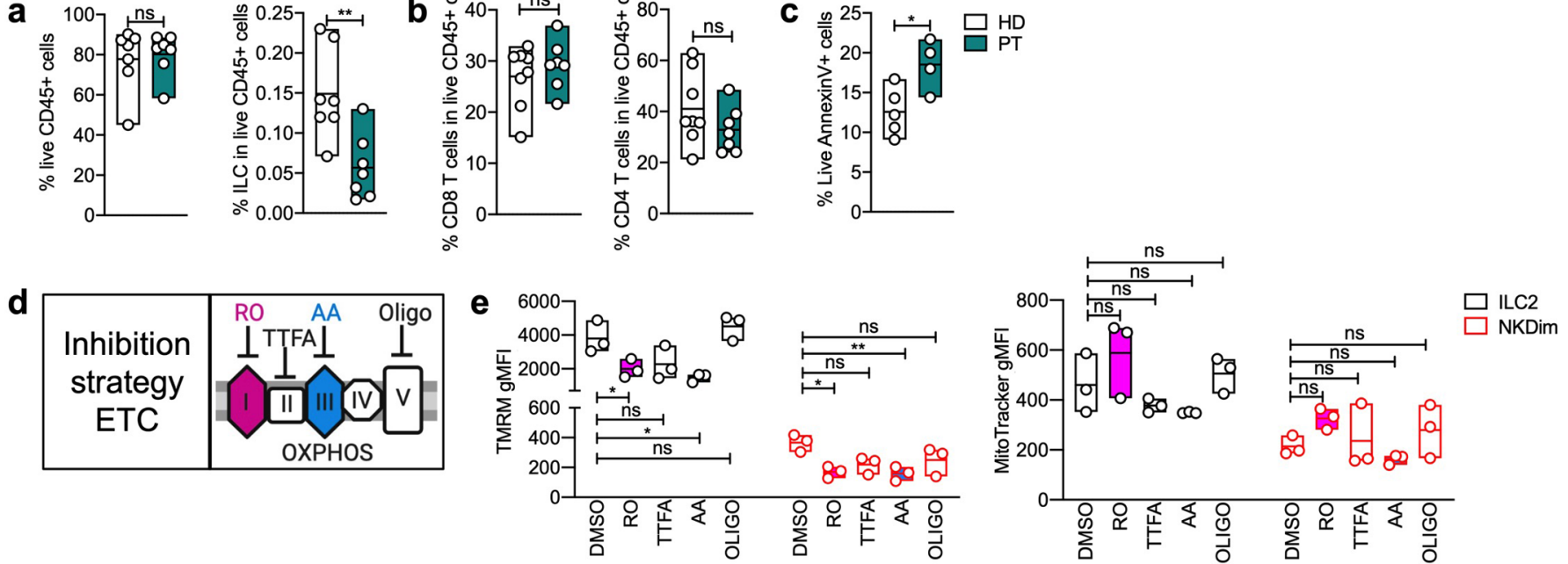

Extended Data Fig. 3 | ILC2 are reduced in patients with mitochondrial diseases. a-c, Comparison of healthy donors (HD, black) and patients with mitochondrial diseases (PT, green). The percentage of live CD45 cells, total ILC (a) CD8 ${ }^{+}$and CD4 $4^{+} \mathrm{T}$ cells (b) and live Annexin- $\mathrm{V}^{+}$cells (c) was measured by FACS (HD=7, PT =7). d-e, Freshly sorted ILC2 and NKDim were cultured with no additional cytokines for 18h in DMSO, Rotenone (1uM), Antimycin A (1uM), TTFA (1uM) or Oligomycin (1uM), as indicated in the inhibition strategy (d). TMRM and MitoTracker gMFI were measured by FACS $(\mathbf{e})(n=3)$. a-c, Each dot represents a donor. $\mathbf{d}-\mathbf{e}$, Data representative of 3 independent experiments with at least 3 donors each. a-c, e, Floating bars indicate the mean, minimum and maximum values within the dataset. Statistics were assessed by two tailed t-test (a-c), and one-way ANOVA with Dunnett correction (e), not significant (ns), $p>0.05 ;{ }^{\star} p<0.05 ;{ }^{* \star} p<0.01 ;{ }^{* \star *} p<0.001 ;{ }^{* \star \star *} p<0.0001$. 
a
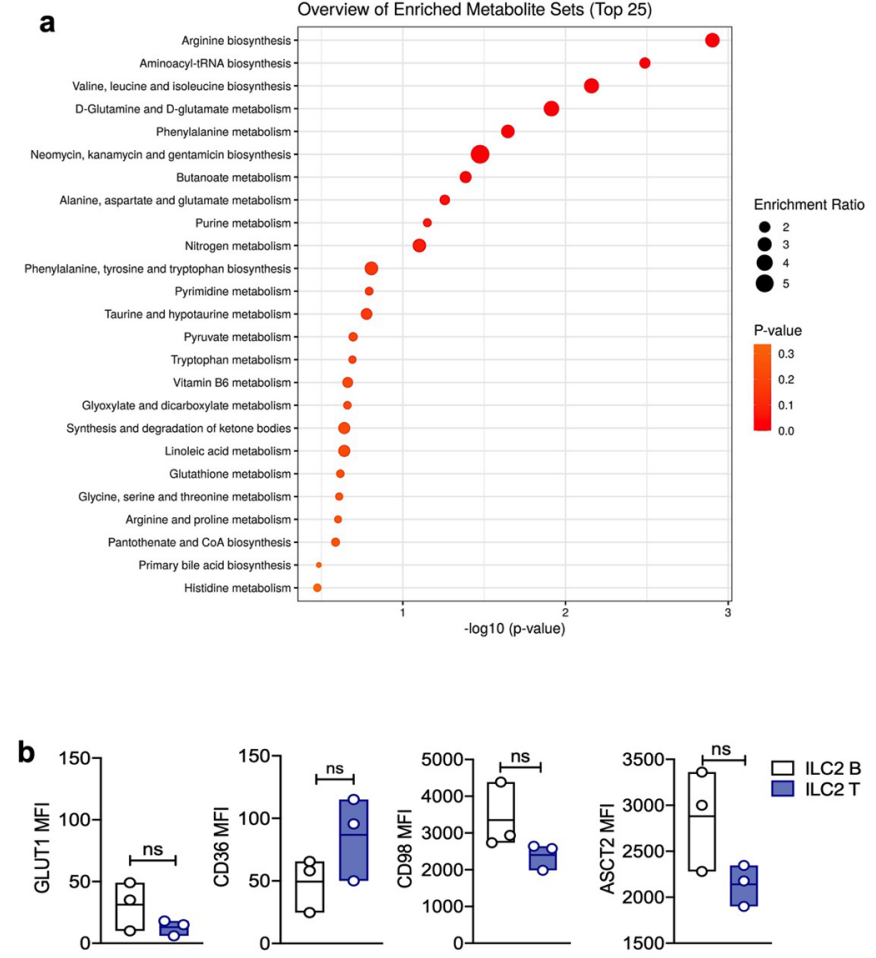
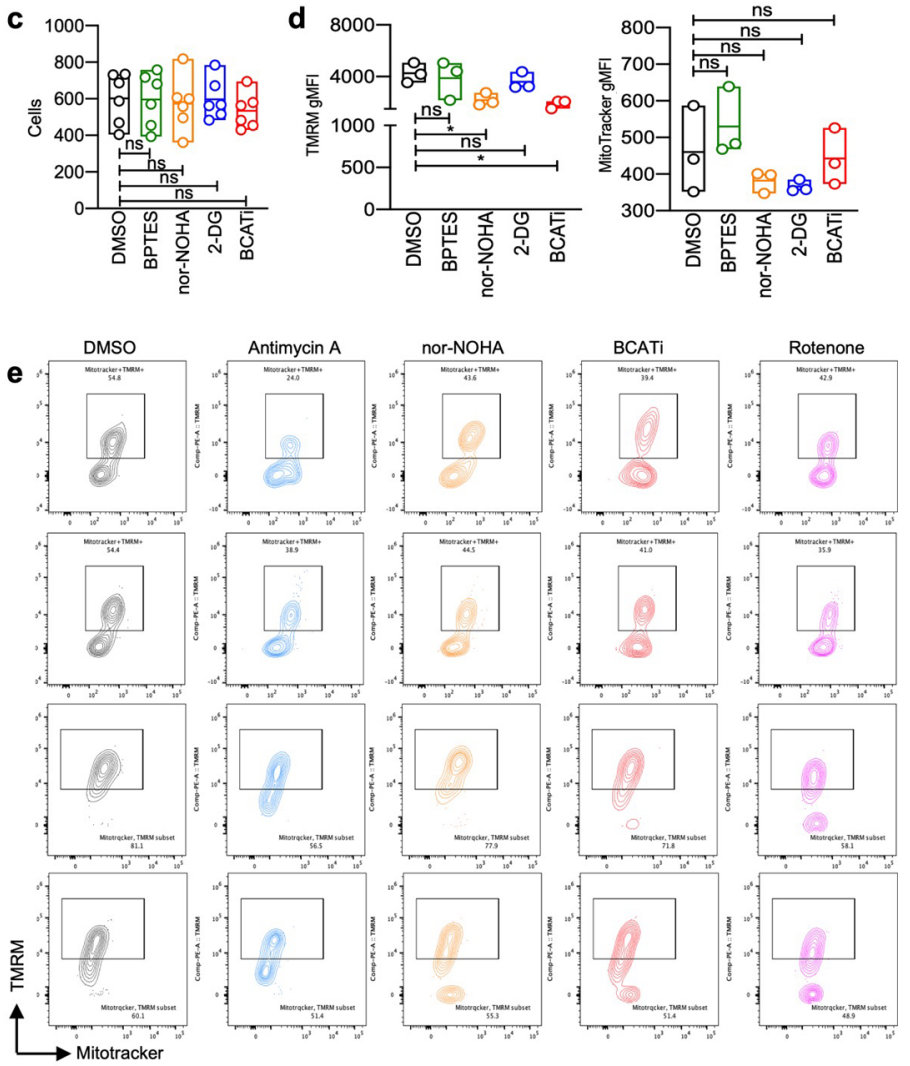

Extended Data Fig. 4 | Branched amino acid and Arginine metabolism support OXPHOS in circulating ILC2. a, Enrichment analysis done by Metaboanalyst. Metabolite intensities were used as input and human metabolome was used as reference. b, Paired blood and tonsil samples were collected from pediatric donors. GLUT1, CD36, CD98 and ASCT2 were measured by FACS ( $n=3)$. c-d, Freshly sorted ILC2 were cultured w/o additional cytokines for 18h in DMSO, nor-NOHA (1uM), BCATi (1uM), BPTES (1uM) or 2DG (1uM). Cell counts (c) $(n=6)$ and TMRM and MitoTracker MFI (d) $(\mathrm{n}=3)$ were measured by FACS. e, FACS analysis of TMRM and Mitotracker staining in ILC2 cultured w/o additional cytokines for 18h in DMSO, Rotenone (1uM), Antimycin A (1uM), nor-NOHA (1uM) and BCATi (1uM). a, Data summarized from 4 donors. A minimum of 2 technical replicates were analyzed per run. b, Data are representative of 2 independent experiments with 3 donors each. c-d, Data are representative of 4 independent experiments with at least 2 donors each. e, Data are shown for 4 donors from 2 independent experiments. b-d, Floating bars indicate the mean, minimum and maximum values within the dataset. Statistics were assessed by two tailed t-test (b) and one-way ANOVA with Dunnett correction (c-d), not significant (ns), p > 0.05; ${ }^{*} p<0.05$. 


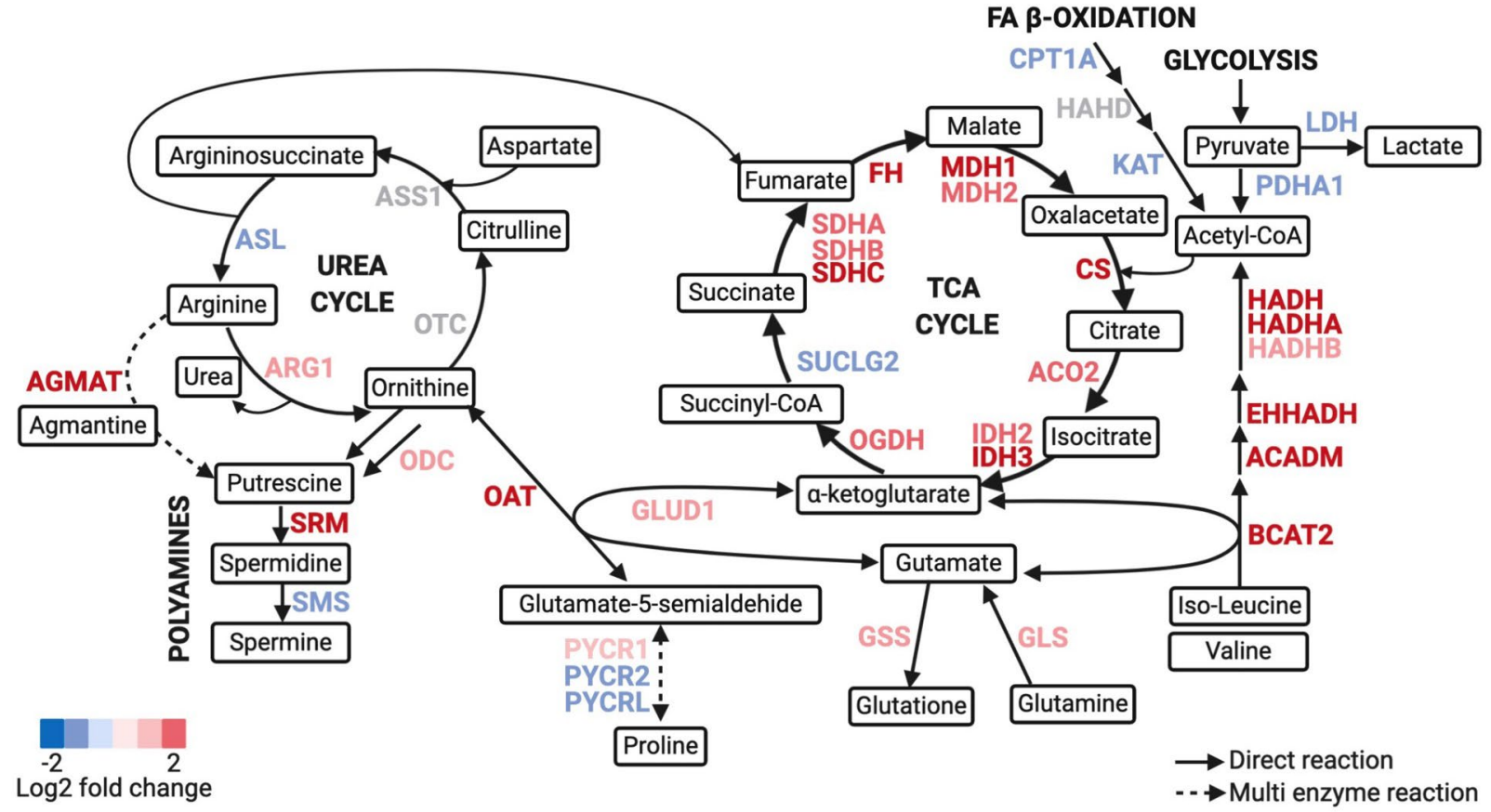

Extended Data Fig. 5 | Branched amino acid and Arginine are used as building blocks to sustain TCA cycle and OXPHOS. Enzyme analysis comparing RNA-seq data to published NK cell dataset for the calculation of the fold change. Data extracted from RNA-seq dataset. Median calculated from values of 3 healthy donors. 
a

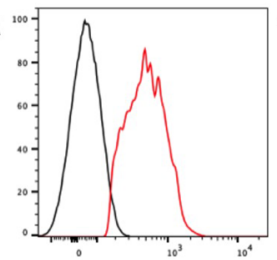

CRTh2

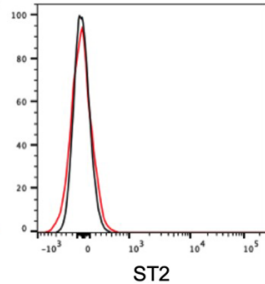

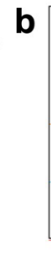

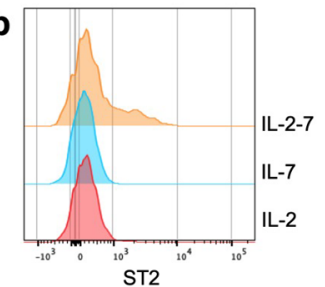

e

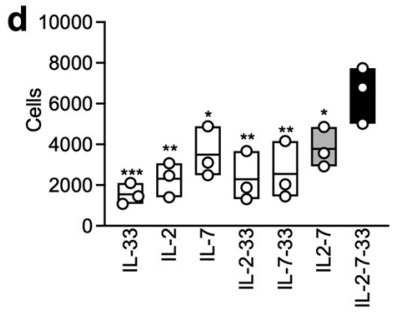

$\mathbf{f}$

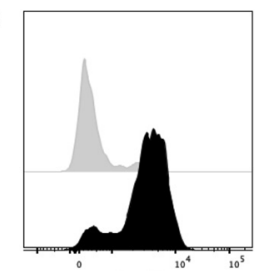

IL-13

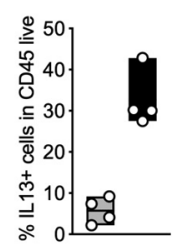

월
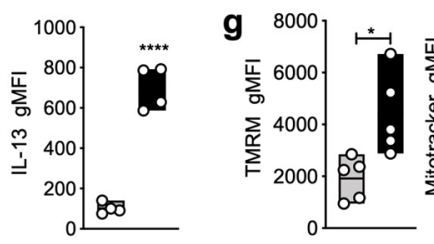
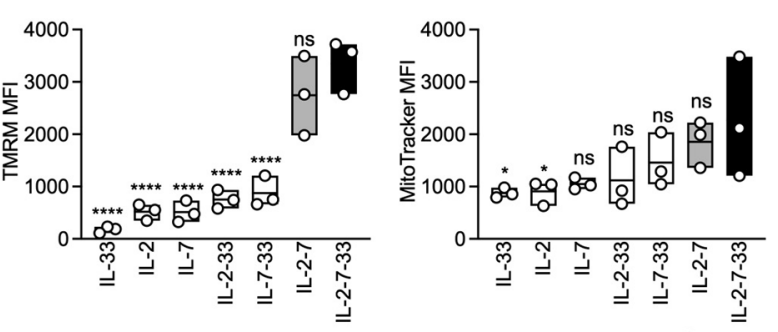

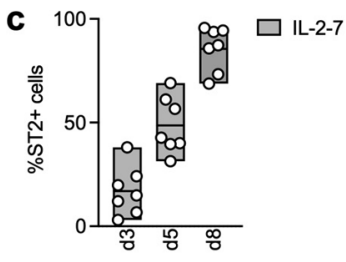

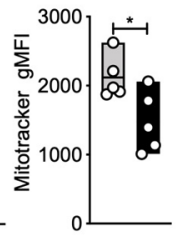

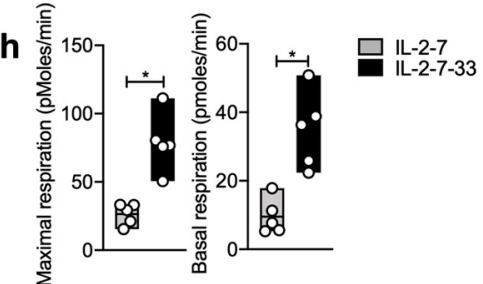

Extended Data Fig. 6 | Activated ILC2 upregulate ST2 and enhance OXPHOS. a, Representative FACS plots of CRTh2 and ST2 in blood ILC2. b, ILC2 were cultured for $18 \mathrm{~h}$ with IL-2, IL-7 or IL-2-7 and levels of ST2 were monitored by FACS. c, ILC2 were expanded for 3, 5 or 7 days in IL-2-7 and ST2 was measured by FACS $(n=7)$. d,e, ILC2 were expanded for 7 days in the indicated cytokine combinations. Cell number was assessed by trypan blue (d), TMRM and MitoTracker gMFI were measured by FACS $(\mathbf{e})(n=3)$. $\mathbf{f}-\mathbf{h}$, ILC2 were expanded for 7 days in IL-2-7 and then IL-33 was added or not for 72h. Levels of IL-13 (f) $(n=4)$, TMRM and MitoTracker $(\mathbf{g})$ were measured at d10 by FACS $(n=5)$. h, Maximal and basal respiration were measured by seahorse upon addition of Glucose, Oligomycin, FCCP and a combination of Rotenone + Antimycin A $(n=5)$. $\mathbf{a}, \mathbf{b}$ Plot representative of 3 different donors. c-e, Data are representative of two experiments with 3 to 4 donors each. $\mathbf{f}$, Data are representative of 3 experiments with at least 3 donors each. $\mathbf{g}$-h, Data are representative of 5 independent experiments with 3 to 5 donors each. $\mathbf{c}-\mathbf{h}$, Floating bars indicate the mean, minimum and maximum values within the dataset. Statistics were assessed by one-way ANOVA with Tukey correction (d,e) and two tailed t-test (f-h), not significant (ns), p $>0.05$; ${ }^{\star} p<0.05$; ${ }^{\star *} \mathrm{p}<0.01 ;{ }^{* \star \star} \mathrm{p}<0.001 ;{ }^{\star \star \star \star} \mathrm{p}<0.0001$. 

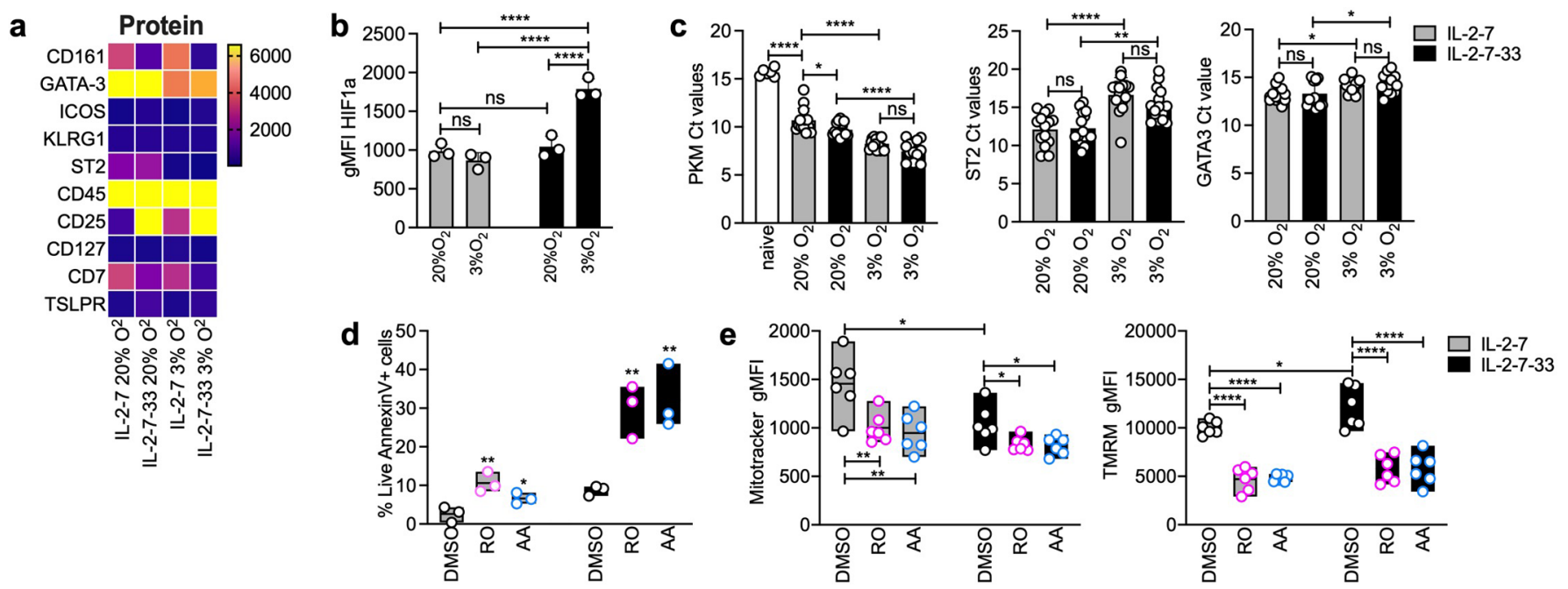

Extended Data Fig. 7 | Oxygen consumption is crucial upon ILC2 activation. a-c, Cells were cultured at 20\% or 3\% oxygen in IL-2-7 or IL-2-7-33 for 5 days. The gMFI of the indicated makers in the heatmap (a) and HIF1a gMFI $(\mathbf{b})$ were analyzed by FACS $(n=3)$. The level of expression of PKM, ST2 and GATA-3 transcripts was measured by Biomark $(\mathbf{c})(n=15)$. d-e, 1uM RO, or 1uM AA or with DMSO were added for 18h to the culture. Annexin V and L/D $(\mathbf{d})(n=3)$, Mitotracker and TMRM MFI $(\mathbf{e})(n=6)$ were analyzed by FACS. $\mathbf{a}$, Data pooled from 3 independent experiments with 3 donors each. $\mathbf{b}$, Data representative of 3 independent experiments. c, Data pooled from 2 independent experiments. d-e, Data are representative of a total of 4 independent experiments with 3 donors each. b-c, Bars are \pm s.e.m.; d-e, Floating bars indicate the mean, minimum and maximum values within the dataset. Statistics were assessed by one-way ANOVA with Tukey correction (b-e), not significant (ns), p $>0.05 ;{ }^{\star} p<0.05 ;{ }^{* \star} p<0.01 ;{ }^{* \star \star} p<0.001 ;{ }^{* \star \star \star} p<0.0001$. 

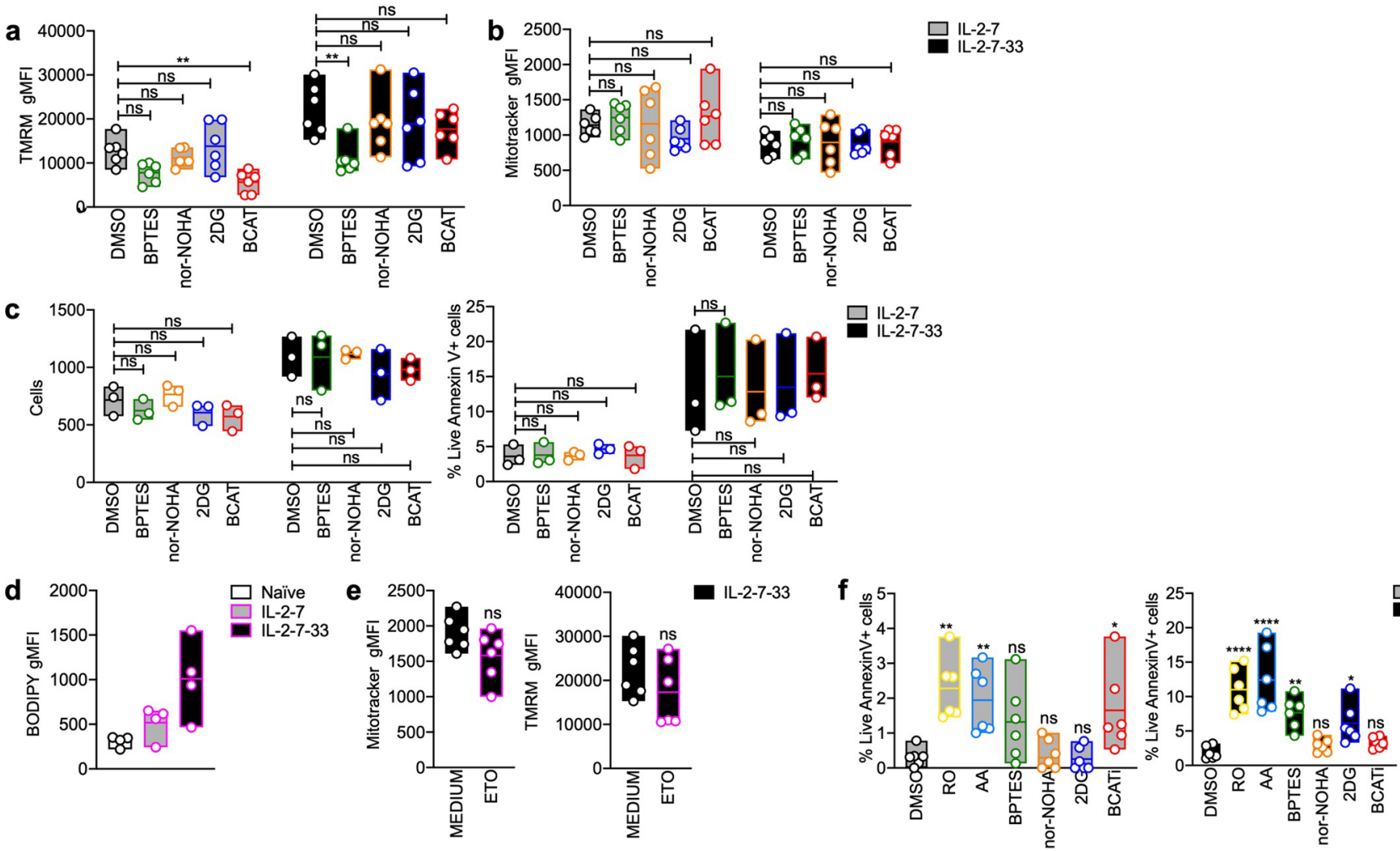

IL-2-7

Extended Data Fig. 8 | Activated ILC2 use different nutrients to sustain proliferation. a-e, ILC2 were expanded for 7 days in IL-2-7 and then IL-33 was added or not for $72 \mathrm{~h}$. Inhibitors or DMSO were added at the concentrations indicated in Fig. 4 for 18h. MitoTracker and TMRM (a-b) ( $\mathrm{n}=6$ ), cell counts and AnnexinV (c) were monitored by FACS $(n=3)$. d-e, Bodipy $(\mathbf{d})(n=4)$, MitoTracker and TMRM $(\mathbf{e})(n=6)$ were analyzed by FACS in naïve, IL-2-7 and IL-2-7-33 stimulated ILC2. f, Cells were cultured for 6 days in IL-2-7 and 1uM RO, 1uM AA, 1uM BPTES, or 1uM 2DG, or 1uM nor-NOHA, or 1uM BCATi or DMSO. Annexin $\vee$ was measured by FACS $(n=6)$. a-b Data are summarizing 2 experiments with 3 donors each. Representative of a total of 4 independent experiments. c-e, Data are representative of 3 independent experiments with at least 3 donors each. f, Data are summarizing 2 experiments with 3 donors each. Representative of a total of 3 independent experiments. Floating bars indicate the mean, minimum and maximum values within the dataset. Statistics were assessed by one-way ANOVA with Dunnett correction (a-d, f) and two tailed t-test in (e), not significant (ns), $p>0.05$; ${ }^{\star} p<0.05$; ${ }^{\star \star} p<0.01 ;{ }^{\star \star \star} p<0.001 ;{ }^{\star \star \star \star} p<0.0001$. 
a

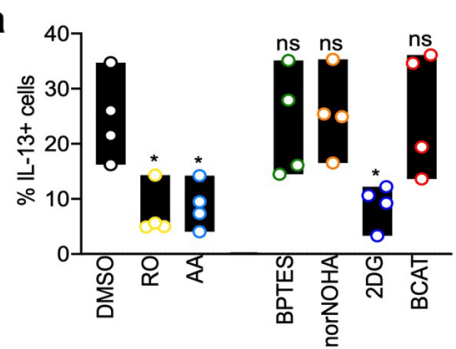

b
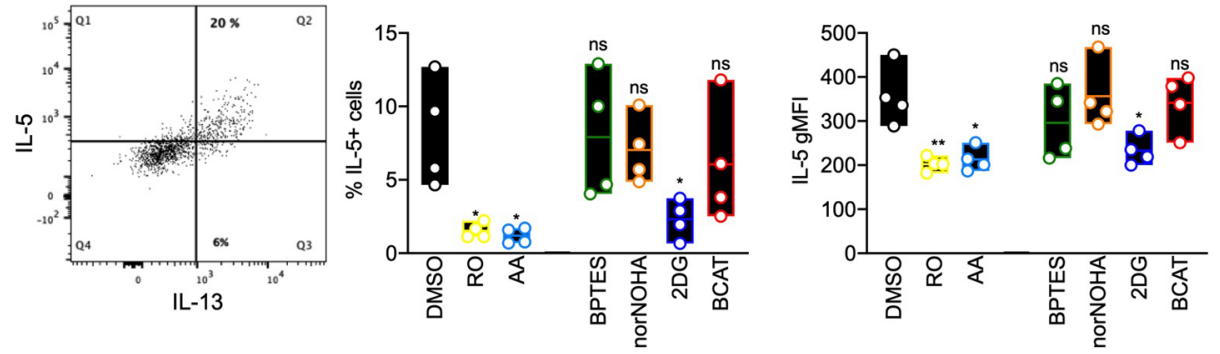
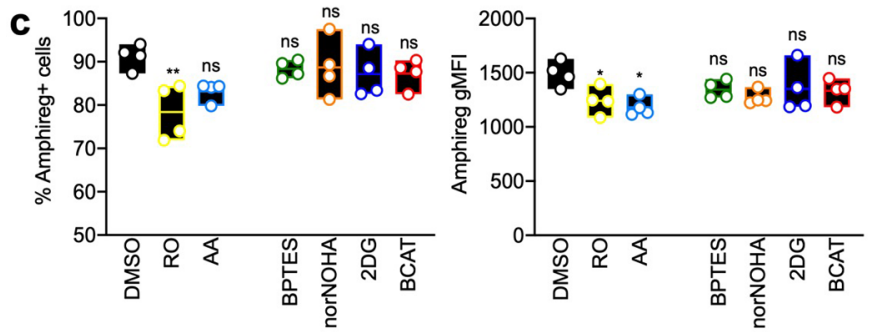

e

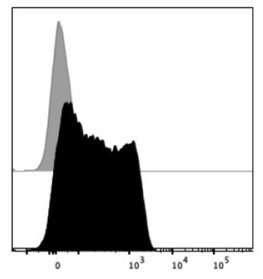

CMXROS
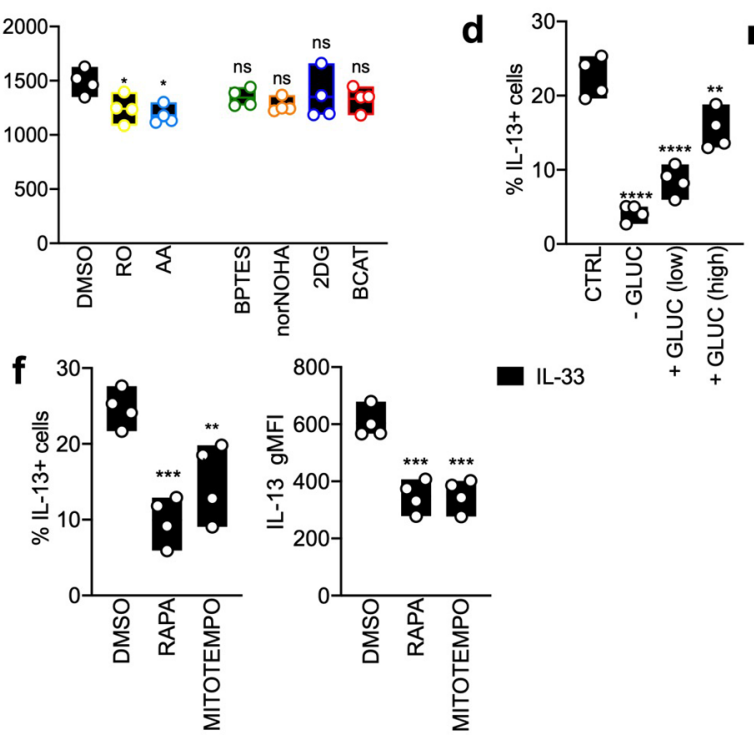

IL-33

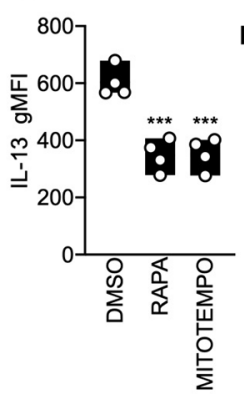

Extended Data Fig. 9 | Activated ILC2 use different pathways to sustain function and proliferation. a-c, ILC2 were expanded in IL-2-7 for 7 days \pm inhibitors as described in (Fig. 4d-e). IL-33 was added for the last 6 hours. Percentage of IL-13+ cells (a) IL-5+ cells and gMFI (b) and amphiregulin+ cells and gMFI (c) were measured by FACS $(n=4)$. d, ILC2 were expanded in IL-2-7 for 7 days and then IL-33 was added for the last 6 hours on cells starved for 1 hour and on glucose add-back condition. Percentage of IL-13+ cells was measured by FACS $(n=4)$. e, ILC2 were expanded for 7 days in IL-2-7 and then IL-33 was added or not for $72 \mathrm{~h}$. Levels of ROS was measured by FACS $(n=4)$. f, ILC2 were expanded in IL-2-7 for 7 days in the presence or not of the inhibitors MitoTempo (1uM) and Rapamycin ( $25 \mathrm{nM})$. IL-33 was added for 6 hours. Percentage of IL-13+ cells was measure by FACS $(n=4)$. a-d, Data are representative of 3 independent experiments with at least 3 donors each. e-f, Data are representative of 2 independent experiments with at least 3 donors each. Floating bars indicate the mean, minimum and maximum values within the dataset. Statistics were assessed by one-way ANOVA with Dunnett correction (a-f), not significant (ns), $p>0.05 ;{ }^{\star} p<0.05 ;{ }^{\star \star} p<0.01 ;{ }^{\star \star \star} p<0.001 ;{ }^{* \star \star \star} p<0.0001$. 


\section{Reporting Summary}

Nature Research wishes to improve the reproducibility of the work that we publish. This form provides structure for consistency and transparency in reporting. For further information on Nature Research policies, see our Editorial Policies and the Editorial Policy Checklist.

\section{Statistics}

For all statistical analyses, confirm that the following items are present in the figure legend, table legend, main text, or Methods section.

$\mathrm{n} / \mathrm{a}$ Confirmed

\ The exact sample size $(n)$ for each experimental group/condition, given as a discrete number and unit of measurement

$\bigotimes$ A statement on whether measurements were taken from distinct samples or whether the same sample was measured repeatedly

$\triangle$ The statistical test(s) used AND whether they are one- or two-sided

Only common tests should be described solely by name; describe more complex techniques in the Methods section.

$\bigotimes$ A description of all covariates tested

\ A description of any assumptions or corrections, such as tests of normality and adjustment for multiple comparisons

$\triangle$ A full description of the statistical parameters including central tendency (e.g. means) or other basic estimates (e.g. regression coefficient)

AND variation (e.g. standard deviation) or associated estimates of uncertainty (e.g. confidence intervals)

For null hypothesis testing, the test statistic (e.g. $F, t, r$ ) with confidence intervals, effect sizes, degrees of freedom and $P$ value noted Give $P$ values as exact values whenever suitable.

Х $\square$ For Bayesian analysis, information on the choice of priors and Markov chain Monte Carlo settings

Х $\square$ For hierarchical and complex designs, identification of the appropriate level for tests and full reporting of outcomes

$\square \bigotimes$ Estimates of effect sizes (e.g. Cohen's $d$, Pearson's $r$ ), indicating how they were calculated

Our web collection on statistics for biologists contains articles on many of the points above.

\section{Software and code}

Policy information about availability of computer code

Data collection no software was used

Data analysis Qlucore Omic Explorer v3, Prism8, FlowJo v10, Matlab R2010b, Hisat (version 0.1.6), HOMER

For manuscripts utilizing custom algorithms or software that are central to the research but not yet described in published literature, software must be made available to editors and

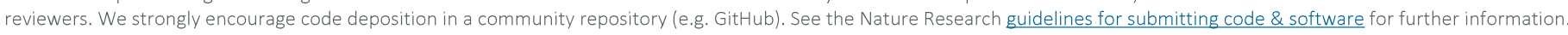

\section{Data}

Policy information about availability of data

All manuscripts must include a data availability statement. This statement should provide the following information, where applicable:

- Accession codes, unique identifiers, or web links for publicly available datasets

- A list of figures that have associated raw data

- A description of any restrictions on data availability

All data generated or analysed during this study are included in this published article (as supplementary information files). RNA-seq datasets have been deposited in the GEO public repository (accession number GSE183669). 
Please select the one below that is the best fit for your research. If you are not sure, read the appropriate sections before making your selection.

\ Life sciences

Behavioural \& social sciences

Ecological, evolutionary \& environmental sciences

For a reference copy of the document with all sections, see nature.com/documents/nr-reporting-summary-flat.pdf

\section{Life sciences study design}

All studies must disclose on these points even when the disclosure is negative.

\begin{tabular}{|c|c|}
\hline Sample size & Sample size was based on donor availability. \\
\hline Data exclusions & No data were excluded from this study. \\
\hline Replication & $\begin{array}{l}\text { Each experiment published in this study has been repeated at least } 2 \text { times (indicated in the figure legends). Crucial experiments were } \\
\text { performed and analyzed interdependently by the first and second authors. }\end{array}$ \\
\hline Randomization & Allocation was random for each experiment. Donors were assigned numbers and shuffled at the beginning of the experiment. \\
\hline Blinding & $\begin{array}{l}\text { confocal microscopy was done in a single blinded fashion. Other experiments ere not done in a blind fashion as the investigators need to } \\
\text { design, conduct and analyze the data, thus they need to know the identification of samples. }\end{array}$ \\
\hline
\end{tabular}

\section{Reporting for specific materials, systems and methods}

We require information from authors about some types of materials, experimental systems and methods used in many studies. Here, indicate whether each material, system or method listed is relevant to your study. If you are not sure if a list item applies to your research, read the appropriate section before selecting a response.

\begin{tabular}{|c|c|c|c|}
\hline \multicolumn{2}{|r|}{ Materials \& experimental systems } & \multicolumn{2}{|c|}{ Methods } \\
\hline $\mathrm{n} / \mathrm{a}$ & Involved in the study & $\mathrm{n} / \mathrm{a}$ & Involved in the study \\
\hline - & Х Antibodies & Х & $\square$ ChIP-seq \\
\hline Х & $\square$ Eukaryotic cell lines & $\square$ & \ Flow cytometry \\
\hline Х & $\square$ Palaeontology and archaeology & $\bigotimes$ & $\square$ MRI-based neuroimaging \\
\hline Х & $\square$ Animals and other organisms & & \\
\hline ᄂ & $\bigotimes$ Human research participants & & \\
\hline Х & $\square$ Clinical data & & \\
\hline Х & $\square$ Dual use research of concern & & \\
\hline
\end{tabular}

\section{Antibodies}

Antibodies used

Surface Glut1 expression was monitored as a function of binding to its ligand, the envelope glycoprotein of the human T lymphotropic virus (HTLV). A recombinant HTLV envelope receptor-binding domain (HRBD) fused to an EGFP coding sequence was used as previously described (Manel et al. 2003). Surface ASCT2 was similarly evaluated; expression was monitored as a function of binding to its ligand, the RD114 envelope glycoprotein of the feline endogenous retrovirus (Laval et al; 2013), fused with a murine Fc tag and revealed with an Alexa-Flour-647-conjugated anti-mouse IgG (Invitrogen).

Antibodies for ILC2 enrichment anti-hCD3 biotin (Clone OKT3, Cat\#13-0037-82, 1/100), anti-hCD4 biotin (RPA-T4, 13-0049-82, 1/100), anti-hCD19 biotin (HIB19, 13-0199-82, 1/100), anti-hCD14 biotin (61D3, 13-0149-82, 1/100), anti-hCD123 biotin (6H6, 13-1239-82, 1/100) and anti-hCD235a biotin (HIR2 GA-R2, 13-9987-82, 1/100) were purchased from eBioscience. Antibodies used for ILC2 sorting anti-hCD3 FITC (UCHT1, 11-0038, 1/100), anti-hCD4 FITC (OKT4, 11-0048, 1/100), anti-hCD5 FITC (UCHT2, 11-0059, 1/100), Anti-haßTCR FITC (IP26, 564451, 1/100), Anti-hySTCR FITC (B1.1, 11-9986, 1/100), anti-hCD127 (IL-7Ra) PE-Cy7 (eBioRDR5, 25-1278-42, 1/50) were purchased from eBioscience. Anti-hCD14 FITC (TUK4, 130-080-701, 0,5/100), anti-hCD19 FITC (LT19, 130-104-650, 0,5/100), Anti-hCD159a (NKG2A) PE (REA110, 130-113-566, 1/500) were purchased from Miltenyi. Anti-hCD294 (CRTH2) Alexa Fluor 647 (BM16, 558042, 1/25), anti-hCD7 BV711 (M-T701, 564018, 1/100) were purchased from BD. Anti-hCD45 AF700 (HI30, 560566, 1/100), anti-hCD94 APC-Fire750 (DX22, 305518, 1/25), anti-hCD117 BV605 (104D2, 313218, 1/50), anti-hCD16 BV650 (3G8, 302042, 1/100), anti-hCD56 BV756 (5.1H11, 362550, 1/100) were purchased from Biolegend. Antibodies for FACS analysis (extracellular and intracellular staining) anti-hAnnexinV BV395 (564871, 1/100), anti-hCD3 BUV737 (UCHT1, 612750, 1/100), anti-hCD5 BUV737 (UCHT2, 612842, 1/100); anti-hCD14 BUV737 (M5E2, 612763, 1/100), anti-hCD19 BUV737 (SJ25C1, 612756, 1/100), anti-hCD45 BV805 (HI30, 612891, 1/200), anti-hIL-13 BV421 (JES10-5A2, 624124, 1/100) were purchased from BD, anti-hST2 APC (hIL33Rcap, 17-9338-42, 1/25) and anti-Amphiregulin (AREG559, 17-5370-42, 1/50) were purchased from ebioscience, anti-hIL-5 (TRFK5, 504311, 1/100) and anti-hHIF1a (546-16,359704, 1/100) were purchased from biolegend.

Antibodies were validated by the supplier. 


\section{Human research participants}

\section{Policy information about studies involving human research participants}

\section{Population characteristics}

Healthy donors included in the study were randomly selected (age, sex). The EFS (Establissement Francais du Sang) did not share information about clinical history or past diagnosis. Mitochondrial disease patients were selected based on the diagnosis (PEO, ADOA, MELAS, PEO+AO), but randomly selected for age and sex. All the patients had genetic mutations as leading cause for mitochondrial disease (described in the table). No clinical history file has been shared by the clinician.

Recruitment

Ethics oversight Healthy donors were randomly recruited by the Establissement Francais du Sang (EFS, Paris). PBMC from mitochondrial disease patients were obtained from Unit of Neurology and Neuromuscular Disorders at "University of Messina".

Note that full information on the approval of the study protocol must also be provided in the manuscript.

\section{Flow Cytometry}

Plots

Confirm that:

\The axis labels state the marker and fluorochrome used (e.g. CD4-FITC).

\The axis scales are clearly visible. Include numbers along axes only for bottom left plot of group (a 'group' is an analysis of identical markers).

\All plots are contour plots with outliers or pseudocolor plots.

\A numerical value for number of cells or percentage (with statistics) is provided.

\section{Methodology}

Sample preparation

Instrument

Software

Cell population abundance

Gating strategy
Isolation of the human peripheral blood mononuclear cells (PBMC) and single cell suspension was achieved by Ficoll-Paque (GE Healthcare) density gradient centrifugation.

Cells were stained with surface antibodies and Flexible Viability Dye efluor 506 (eBioscience) in PBS 2\%FCS for 30 minutes on ice. For experiments involving intracellular staining of cytokines, cells were stimulated for 6 hours with cytokines and during the last 3 hours Golgi Plug and Golgi Stop (BD) were added to the cultures. Cells were washed with PBS and fixed/ permeabilized for 45 minutes at RT by Cytofix/Cytoperm kit (BD). Intracellular staining was performed at RT for 30 minutes in the dark. AnnexinV staining was performed using the Annexin V binding buffer (BD). Samples were acquired at the LSRFortessa (BD) and analyzed by FlowJ10.7.1 (Tree Star). For cell sorting PBMC were depleted of T cell, B cells, pDC, monocytes and erythrocytes by labeling with biotin conjugated antibodies, followed by anti-biotin microbeads and AutoMACS separation (Miltenyi) according to manufacturer's instruction. Cells were sorted in bulks to a purity of $\geq 99 \%$ (FACSAria II; BD).

LSRFortessa (BD), (FACSAria II; BD)

FlowJo, Diva

Cells were sorted in bulks to a purity of $\geq 99 \%$ (screened by FACS) or as single cell index sorting (FACSAria II score)

ILCs Gating strategy: Live CD45+ CD7+ Lin- CD94- NKG2A- CD56- CD16- CD127+ CRTh2+ (ILC2) and Live CD45+ CD7+ LinCD94+ CD16+ (NKDim).

$\bigotimes$ Tick this box to confirm that a figure exemplifying the gating strategy is provided in the Supplementary Information. 